Article

\title{
Banach-Space Operators Acting on Semicircular Elements Induced by $p$-Adic Number Fields over Primes $p$
}

\section{Ilwoo Cho}

Department of Mathematics and Statistics, Saint Ambrose University, 421 Ambrose Hall, 518 W. Locust St., Davenport, IA 52803, USA; choilwoo@sau.edu

Received: 28 April 2019; Accepted: 23 May 2019; Published: 01 June 2019

Abstract: In this paper, we study certain Banach-space operators acting on the Banach *-probability space $\left(\mathbb{L S}, \tau^{0}\right)$ generated by semicircular elements $\Theta_{p, j}$ induced by $p$-adic number fields $\mathbb{Q}_{p}$ over the set $\mathcal{P}$ of all primes $p$. Our main results characterize the operator-theoretic properties of such operators, and then study how $\left(\mathbb{L} \mathbb{S}, \tau^{0}\right)$.

Keywords: free probability; $p$-adic number fields; weighted-semicircular elements; semicircular elements; the semicircular adelic filterization; shifts on $\mathcal{P} \times \mathbb{Z}$; free homomorphisms; prime-integer-shift operators

MSC: 11G15; 11R47; 11R56; 46L10; 46L54; 47L30; 47L55

\section{Introduction}

The main purposes of this paper are to study certain Banach-space operators acting on the Banach *-algebra $\mathbb{L} \mathbb{S}$ generated by mutually free, infinitely-many semicircular elements induced by measurable functions on the $p$-adic number fields $\mathbb{Q}_{p}$, for primes $p$ in the set $\mathcal{P}$ of all primes. By regarding the Banach *-algebra $\mathbb{L} \mathbb{S}$ as a Banach space, we construct-and-consider certain Banach-space operators acting on $\mathbb{L} \mathbb{S}$. In particular, we are interested in the case where these operators are generated by certain $*$-homomorphisms in the homomorphism semigroup Hom $(\mathbb{L} \mathbb{S})$, induced by shifting processes on the Cartesian product set $\mathbb{P}=\mathcal{P} \times \mathbb{Z}$, where $\mathbb{Z}$ is the set of all integers. Note that our shifting processes here are well-defined by understanding the sets $\mathcal{P}$ and $\mathbb{Z}$ as totally ordered sets (in short, TOsets) under the usual inequality $(\leq)$.

Our main results categorize such Banach-space operators as in the usual Hilbert-space operator (spectral) theory. Artificially, but naturally, we study self-adjointness, projection-property, normality, isometry-property, and unitarity of these operators acting on the semicircular law (see Section 13 below). In addition, they show that some of such Banach-space operators preserves the free probability on $\mathbb{L} \mathbb{S}$, and hence the semicircular law (which is the free distributions of the free generators of $\mathbb{L} \mathbb{S}$ ) is preserved by the action of the operators; meanwhile, some of such operators distort the semicircular law, whose distortions are characterizable (see Section 14 below).

\subsection{Preview and Motivation}

Connections between primes and operators have been considered in different approaches (e.g., [1-7]). For instance, we consider relations between analysis on $\mathbb{Q}_{p}$, and (weighted-) semicircular elements, in [8-10]. In addition, the main results of them are globalized in [3], i.e., connections between 
analysis on the finite Adelic ring, and (weighted-)semicircular elements are considered there with help of Adelic Banach-operator theory of [2].

In [10], the author and Jorgensen studied (weighted-)semicircular elements induced by measurable functions on $\mathbb{Q}_{p}$, for $p \in \mathcal{P}$, by using free-probabilistic models and terminology. It shows that the analysis lets us have certain operators having their statistical distributions, the semicircular(-like) law(s). In [8], the author extended the constructions of (weighted-)semicircular elements of [10] under (free-probabilistic) free product (e.g., [11-15]). It showed that the statistical information of [10] (from free-probabilistic models) are indeed well-determined free-probability-theoretically.

As an application of [8], we studied corresponding free stochastic calculus in [9]. In addition, the main results of [8] are globalized in [3], by extending (local) $p$-adic settings to (global) Adelic settings, with help of [2].

Independent from the above series of research, the author and Jorgensen considered the density of primes in an arbitrarily given interval of the set $\mathbb{R}$ of real numbers, and the semicircular elements induced by $\left\{\mathbb{Q}_{p}\right\}_{p \in \mathcal{P}}$, in [16]. Interestingly, it is shown that such a density deform the the semicircular law induced by $\left\{\mathbb{Q}_{p}\right\}_{p \in \mathcal{P}}$.

\subsection{Overview}

This paper consists of four main parts; the first part is devoted to reconsider (weighted-)semicircular elements induced by $\mathbb{Q}_{p}$, in short Section 2 through Section 7. In the second part, we construct the Banach $*$-probability space $\mathbb{L} \mathbb{S}$ generated by our semicircular elements, and study operator-algebraic, and free-probabilistic properties of $\mathbb{L} \mathbb{S}$. In addition, then $*$-homomorphisms acting on $\mathbb{L} \mathbb{S}$, induced by certain shifting processes on the set $\mathcal{P}$ of primes, and those on the set $\mathbb{Z}$ of integers, are defined-and-studied. In particular, it is shown that these morphisms are free-homomorphisms on $\mathbb{L} \mathbb{S}$ (see Sections 8-10). In the third part, by applying the free-homomorphisms, we construct the commutative monoid $\sigma(\mathbb{L} \mathbb{S})$ acting on $\mathbb{L} \mathbb{S}$, as an algebraic sub-structure of the homomorphism semigroup Hom $(\mathbb{L} \mathbb{S})$ of $\mathbb{L} \mathbb{S}$ (see Sections 11 and 12). Finally, we study Banach-space operators generated by $\sigma(\mathbb{L S})$, contained in the operator space $B(\mathbb{L S}$ ) (in the sense of [2]), and investigate operator-theoretic properties of them (see Section 13), and then consider how such operators deform the original free-distributional data on $\mathbb{L} \mathbb{S}$ (see Section 14).

\section{Preliminaries}

In this section, we briefly mention about backgrounds of our proceeding works.

\subsection{Free Probability}

Free probability is the noncommutative operator-algebraic version of classical measure theory (including probability theory) and statistical analysis (e.g., [11-15]). The classical independence is replaced by the freeness, by replacing measures on sets to linear functionals on algebras. It has various applications not only in pure mathematics (e.g., [17-19]), but also in related topics (e.g., [8-10,20-23]).

In particular, we will use combinatorial free probability of Speicher (e.g., [11-13]). In text, without introducing detailed definitions and combinatorial backgrounds, free moments and free cumulants of operators will be computed. In addition, we use free product of $*$-probability spaces, without precise introduction.

\subsection{Analysis on $\mathbb{Q}_{p}$}

For more about $p$-adic number fields, and corresponding analyses, see [24]. We will use the same definitions, terminology, and notations of [24]. Let $\mathbb{Q}_{p}$ be the $p$-adic number fields for $p \in \mathcal{P}$. Recall that $\mathbb{Q}_{p}$ are the maximal $p$-norm-topology closures in the normed space $\left(\mathbb{Q},|\cdot|_{p}\right)$ of all rational numbers, where $|\cdot|_{p}$ are the non-Archimedean norms, called $p$-norms on $\mathbb{Q}$, for all $p \in \mathcal{P}$. 
For any fixed $p \in \mathcal{P}$, the Banach space $\mathbb{Q}_{p}$ forms a field algebraically under the $p$-adic addition and the $p$-adic multiplication of [24], i.e., $\mathbb{Q}_{p}$ is a Banach field.

In addition, such a Banach field $\mathbb{Q}_{p}$ is understood as a measure space

$$
\mathbb{Q}_{p}=\left(\mathbb{Q}_{p}, \sigma\left(\mathbb{Q}_{p}\right), \mu_{p}\right),
$$

equipped with the left-and-right additive-invariant Haar measure $\mu_{p}$ on the $\sigma$-algebra $\sigma\left(\mathbb{Q}_{p}\right)$, satisfying that

$$
\mu_{p}\left(\mathbb{Z}_{p}\right)=1
$$

where $\mathbb{Z}_{p}$ is the unit disk of $\mathbb{Q}_{p}$,

$$
\mathbb{Z}_{p} \stackrel{\text { def }}{=}\left\{x \in \mathbb{Q}_{p}:|x|_{p} \leq 1\right\} \text { in } \mathbb{Q}_{p}
$$

consisting of all $p$-adic integers of $\mathbb{Q}_{p}$, for all $p \in \mathcal{P}$ (e.g., [24,25]).

As a topological space, the $p$-adic number field $\mathbb{Q}_{p}$ contains its basis elements

$$
U_{k}=p^{k} \mathbb{Z}_{p}=\left\{p^{k} x \in \mathbb{Q}_{p}: x \in \mathbb{Z}_{p}\right\},
$$

satisfying $\mu_{p}\left(U_{k}\right)=\frac{1}{p^{k}}$, for all $k \in \mathbb{Z}$. (e.g., [24]).

By regarding $\mathbb{Q}_{p}$ as a measure space, one can establish a $*$-algebra $\mathcal{M}_{p}$ over $\mathbb{C}$ as a $*$-algebra,

$$
\mathcal{M}_{p}=\mathbb{C}\left[\left\{\chi_{S}: S \in \sigma\left(\mathbb{Q}_{p}\right)\right\}\right]
$$

consisting of $\mu_{p}$-measurable functions $f$,

$$
f=\sum_{S \in \sigma\left(\mathbb{Q}_{p}\right)} t_{S} \chi_{S} \quad\left(t_{S} \in \mathbb{C}\right),
$$

where the sum $\sum$ is the finite sum, and $\chi_{S}$ are the usual characteristic functions of $S$.

On $\mathcal{M}_{p}$, one can naturally define a linear functional $\varphi_{p}$ by the integral, i.e.,

$$
\varphi_{p}(f)=\int_{\mathbb{Q}_{p}} f d \mu_{p}, \forall f \in \mathcal{M}_{p} .
$$

Remark 1. By (2), this linear functional $\varphi_{p}$ is unbounded on $\mathcal{M}_{p}$. Indeed, the algebra $\mathcal{M}_{p}$ contains its unity (or the multiplication-identity, or the unit) $\chi_{\mathbb{Q}_{p}}$, satisfying that

$$
\varphi_{p}\left(\chi_{\mathbb{Q}_{p}}\right)=\mu_{p}\left(\chi_{\mathbb{Q}_{p}}\right)=\infty .
$$

Define now subsets $\partial_{k}$ of $\mathbb{Q}_{p}$ by

$$
\partial_{k}=U_{k} \backslash U_{k+1}, \text { for all } k \in \mathbb{Z},
$$

where $U_{k}$ are the basis elements (1) of $\mathbb{Q}_{p}$. We call these $\mu_{p}$-measurable subsets $\partial_{k}$ of (3), the $k$-th boundaries (of $U_{k}$ ), for all $k \in \mathbb{Z}$. By the basis property of the subsets $\left\{U_{k}\right\}_{k \in \mathbb{Z}}$, one obtains that

$$
\mathbb{Q}_{p}=\underset{k \in \mathbb{Z}}{\sqcup} \partial_{k}
$$

where $\sqcup$ means the disjoint union. In addition, one has

$$
\mu_{p}\left(\partial_{k}\right)=\mu_{p}\left(U_{k}\right)-\mu_{p}\left(U_{k+1}\right)=\frac{1}{p^{k}}-\frac{1}{p^{k+1}},
$$

for all $k \in \mathbb{Z}$.

Note that, by (4), if $S \in \sigma\left(\mathbb{Q}_{p}\right)$, then there exists a subset $\Lambda_{S}$ of $\mathbb{Z}$, such that 


$$
\Lambda_{S}=\left\{j \in \mathbb{Z}: S \cap \partial_{j} \neq \varnothing\right\}
$$

Proposition 1. Let $S \in \sigma\left(\mathbb{Q}_{p}\right)$, and let $\chi_{S} \in \mathcal{M}_{p}$. Then, there exists $r_{j} \in \mathbb{R}$, such that

$$
0 \leq r_{j} \leq 1 \text { in } \mathbb{R}, \text { forall } \in \Lambda_{S},
$$

and

$$
\varphi_{p}\left(\chi_{S}\right)=\int_{\mathbb{Q}_{p}} \chi_{S} d \mu_{p}=\sum_{j \in \Lambda_{S}} r_{j}\left(\frac{1}{p^{j}}-\frac{1}{p^{j+1}}\right)
$$

where $\Lambda_{S}$ is in the sense of (6).

Proof. The computation (7) is shown by (5). See [10] for details.

\section{Free-Probabilistic Models on $\mathcal{M}_{p}$}

Throughout this section, fix a prime $p \in \mathcal{P}$, and the corresponding $p$-adic number field $\mathbb{Q}_{p}$, and let $\mathcal{M}_{p}$ be the $*$-algebra induced by $\mathbb{Q}_{p}$. In this section, let us establish a suitable free-probabilistic model on $\mathcal{M}_{p}$, implying the number-theoretic data.

Let $U_{k}=p^{k} \mathbb{Z}_{p}$ be the basis elements (1), and $\partial_{k}$, their boundaries (3) of $\mathbb{Q}_{p}$, i.e.,

$$
\partial_{k}=U_{k} \backslash U_{k+1}, \text { for all } k \in \mathbb{Z} .
$$

Recall the linear functional $\varphi_{p}$ of (2) on $\mathcal{M}_{p}$,

$$
\varphi_{p}(f)=\int_{\mathbb{Q}_{p}} f d \mu_{p}, \text { for all } f \in \mathcal{M}_{p} .
$$

Then, by (7) and (9), one obtains that

$$
\varphi_{p}\left(\chi_{U_{j}}\right)=\frac{1}{p^{j}}, \text { and } \varphi_{p}\left(\chi_{\partial_{j}}\right)=\frac{1}{p^{j}}-\frac{1}{p^{j+1}},
$$

since

$$
\Lambda_{U_{j}}=\{k \in \mathbb{Z}: k \geq j\}, \text { and } \Lambda_{\partial_{j}}=\{j\},
$$

for all $j \in \mathbb{Z}$.

Let $\partial_{k}$ be the $k$-th boundaries (8) of $\mathbb{Q}_{p}$, for all $k \in \mathbb{Z}$. Then, for $k_{1}, k_{2} \in \mathbb{Z}$, one obtains that

$$
\chi_{\partial_{k_{1}}} \chi_{\partial_{k_{2}}}=\chi_{\partial_{k_{1}} \cap \partial_{k_{2}}}=\delta_{k_{1}, k_{2}} \chi_{\partial_{k_{1}}}
$$

where $\delta$ is the Kronecker delta, and, hence,

$$
\begin{aligned}
\varphi_{p}\left(\chi_{\partial_{k_{1}}} \chi_{\partial_{k_{2}}}\right) & =\delta_{k_{1}, k_{2}} \varphi_{p}\left(\chi_{\partial_{k_{1}}}\right) \\
& =\delta_{k_{1}, k_{2}}\left(\frac{1}{p^{k_{1}}}-\frac{1}{p^{k_{1}+1}}\right),
\end{aligned}
$$

by Labels (10) and (11).

If $S_{1}, S_{2} \in \sigma\left(\mathbb{Q}_{p}\right)$, then it is not difficult to have

$$
\chi_{S_{1}} \chi_{S_{2}}=\sum_{j \in \Lambda_{S_{1}, S_{2}}} \chi_{\left(S_{1} \cap S_{2}\right) \cap \partial_{j}}
$$

where 


$$
\Lambda_{S_{1}, S_{2}}=\Lambda_{S_{1}} \cap \Lambda_{S_{2}}
$$

because $\partial_{k} \cap \partial_{j}=\varnothing$, the empty set, whenever $k \neq j$ in $\mathbb{Z}$.

Proposition 2. Let $S_{l} \in \sigma\left(\mathbb{Q}_{p}\right)$, and let $\chi_{S_{l}} \in\left(\mathcal{M}_{p}, \varphi_{p}\right)$, for $l=1, \ldots, N$, for $N \in \mathbb{N}$. Let

$$
\Lambda_{S_{1}, \ldots, S_{N}}=\bigcap_{l=1}^{N} \Lambda_{S_{l}} \text { in } \mathbb{Z},
$$

where $\Lambda_{S_{l}}$ are in the sense of Label (7), for $l=1, \ldots, N$. Then, there exists $r_{j} \in \mathbb{R}$, such that

$$
\begin{aligned}
& 0 \leq r_{j} \leq 1 \text { in } \mathbb{R}, \\
& \text { and } \\
& \varphi_{p}\left(\prod_{l=1}^{N} \chi_{S_{l}}\right)=\sum_{j \in \Lambda_{S_{1}, \ldots, S_{N}}} r_{j}\left(\frac{1}{p^{j}}-\frac{1}{p^{j+1}}\right) .
\end{aligned}
$$

Proof. The Formula (14) is proven by (7), (12) and (13).

\section{Representations of $\left(\mathcal{M}_{p}, \varphi_{p}\right)$}

Fix a prime $p \in \mathcal{P}$. Construct the $L^{2}$-Hilbert space,

$$
H_{p} \stackrel{\text { def }}{=} L^{2}\left(\mathbb{Q}_{p}, \sigma\left(\mathbb{Q}_{p}\right), \mu_{p}\right)=L^{2}\left(\mathbb{Q}_{p}\right),
$$

over $\mathbb{C}$, equipped with its inner product $<_{1}>_{2}$,

$$
\left\langle f_{1}, f_{2}\right\rangle_{2} \stackrel{\text { def }}{=} \int_{\mathbb{Q}_{p}} f_{1} f_{2}^{*} d \mu_{p},
$$

for all $f_{1}, f_{2} \in H_{p}$, inducing the $L^{2}$-norm,

$$
\|f\|_{2} \stackrel{\text { def }}{=} \sqrt{\langle f, f\rangle_{2}} \text {, for all } f \in H_{p},
$$

where $<_{,}>_{2}$ is the inner product (16) on $H_{p}$. Remark that the unity $\chi_{\mathbb{Q}_{p}} \in \mathcal{M}_{p}$ is not contained in $H_{p}$, since $\left\|\chi_{\mathbb{Q}_{p}}\right\|_{p}=\infty$.

Definition 1. We call the Hilbert space $H_{p}$ of (15), the p-adic Hilbert space.

By Definition 1 of the $p$-adic Hilbert space $H_{p}$, our $*$-algebra $\mathcal{M}_{p}$ acts on $H_{p}$, via an algebra-action $\alpha^{p}$,

$$
\alpha^{p}(f)(h)=f h, \text { for all } h \in H_{p},
$$

for all $f \in \mathcal{M}_{p}$. For example, the morphism $\alpha^{p}$ of (17) is a $*$-homomorphism from $\mathcal{M}_{p}$ to the operator algebra $B\left(H_{p}\right)$ of all operators on $H_{p}$. Indeed, for any $f \in \mathcal{M}_{p}$, the image $\alpha^{p}(f)$ is a well-defined bounded multiplication operator on $H_{p}$ with its symbol $f$, satisfying

$$
\alpha^{p}\left(f_{1} f_{2}\right)=\alpha^{p}\left(f_{1}\right) \alpha^{p}\left(f_{2}\right), \forall f_{1}, f_{2} \in \mathcal{M}_{p},
$$

and

$$
\left(\alpha^{p}(f)\right)^{*}=\alpha^{p}\left(f^{*}\right), \forall f \in \mathcal{M}_{p}
$$

denote $\alpha^{p}(f)$ by $\alpha_{f}^{p}$, for all $f \in \mathcal{M}_{p}$. In addition, for convenience, denote $\alpha_{\chi_{S}}^{p}$ simply by $\alpha_{S}^{p}$, for all $S \in \sigma\left(\mathbb{Q}_{p}\right)$.

Note that the unity $\chi_{\mathbb{Q}_{p}}$ of $\mathcal{M}_{p}$ act on $H_{p}$ (under the action $\alpha^{p}$ of (17)) as the identity operator $1_{H_{p}}$ of the operator algebra $B\left(H_{p}\right)$, in the sense that: 


$$
\alpha_{\mathbb{Q}_{p}}^{p}(f)=\chi_{\mathbb{Q}_{p}} f=f=1_{H_{p}}(f), \text { for all } f \in H_{p}
$$

Proposition 3. (See [10]) The pair $\left(H_{p}, \alpha^{p}\right)$ is a well-determined Hilbert-space representation of $\mathcal{M}_{p}$.

Definition 2. Let $B\left(H_{p}\right)$ be the operator algebra consisting of all operators on $H_{p}$. Define the $C^{*}$-subalgebra $M_{p}$ of $B\left(H_{p}\right)$ by

$$
\left.M_{p} \stackrel{\text { def }}{=} \overline{\alpha^{p}\left(\mathcal{M}_{p}\right)}\|\cdot\|=\overline{\mathbb{C}\left[\alpha_{f}^{p}: f \in \mathcal{M}_{p}\right]}\right]^{\|\cdot\|}
$$

where $\bar{X}^{\|\cdot\|}$ mean the operator-norm closures of subsets $X$ of $B\left(H_{p}\right)$. This $C^{*}$-algebra $M_{p}$ of $(18)$ is called the p-adic $C^{*}$-algebra of $\left(\mathcal{M}_{p}, \varphi_{p}\right)$.

\section{Free-Probabilistic Models on $M_{p}$}

Throughout this section, let's fix a prime $p \in \mathcal{P}$. Let $M_{p}$ be the $p$-adic $C^{*}$-algebra of (18). Define a linear functional $\varphi_{j}^{p}: M_{p} \rightarrow \mathbb{C}$ by a linear morphism,

$$
\varphi_{j}^{p}(a) \stackrel{\text { def }}{=}\left\langle a\left(\chi_{\partial_{j}}\right), \chi_{\partial_{j}}\right\rangle_{2}, \forall a \in M_{p},
$$

for all $j \in \mathbb{Z}$, where $<_{,}>_{2}$ is the inner product (16) on the $p$-adic Hilbert space $H_{p}$ of (15).

Note that these linear functionals $\varphi_{j}^{p}$ are bounded on $M_{p}$ because

$$
\begin{aligned}
\varphi_{j}^{p}\left(\alpha_{\mathbb{Q}_{p}}^{p}\right) & =\left\langle\chi_{\mathbb{Q}_{p}} \chi_{\partial_{j}}, \chi_{\partial_{j}}\right\rangle_{2}=\left\langle\chi_{\mathbb{Q}_{p} \cap \partial_{j}}, \chi_{\partial_{j}}\right\rangle_{2} \\
& =\int_{\mathbb{Q}_{p}} \chi_{\partial_{j}} d \mu_{p} \\
& =\mu_{p}\left(\chi_{\partial_{j}}\right)=\frac{1}{p^{j}}-\frac{1}{p^{j+1}}<\infty,
\end{aligned}
$$

for any arbitrarily fixed $j \in \mathbb{Z}$.

Definition 3. Let $j \in \mathbb{Z}$, and let $\varphi_{j}^{p}$ be the linear functional (19) on the $p$-adic $C^{*}$-algebra $M_{p}$. Then, the pair $\left(M_{p}, \varphi_{j}^{p}\right)$ is said to be the $j$-th $p$-measure space.

Now, fix $j \in \mathbb{Z}$, and take the $j$-th $p$-measure space $\left(M_{p}, \varphi_{j}^{p}\right)$. For $S \in \sigma\left(\mathbb{Q}_{p}\right)$, and an element $\alpha_{S}^{p} \in$ $M_{p}$, one has that

$$
\varphi_{j}^{p}\left(\alpha_{S}^{p}\right)=\left\langle\alpha_{S}^{p}\left(\chi_{\partial_{j}}\right), \chi_{\partial_{j}}\right\rangle_{2}=r_{S}\left(\frac{1}{p^{j}}-\frac{1}{p^{j+1}}\right),
$$

for some $0 \leq r_{S} \leq 1$ in $\mathbb{R}$.

Proposition 4. Let $\partial_{k}$ be the $k$-th boundaries (8) of $\mathbb{Q}_{p}$, for all $k \in \mathbb{Z}$. Then,

$$
\varphi_{j}^{p}\left(\left(\alpha_{\partial_{k}}^{p}\right)^{n}\right)=\delta_{j, k}\left(\frac{1}{p^{j}}-\frac{1}{p^{j+1}}\right)
$$

for all $n \in \mathbb{N}$, for $k \in \mathbb{Z}$.

Proof. Note that $\alpha_{\partial_{k}}^{p}$ are projections in $M_{p}$ in the sense that:

$$
\left(\alpha_{\partial_{k}}^{p}\right)^{2}=\alpha_{\partial_{k}}^{p}=\left(\alpha_{\partial_{k}}^{p}\right)^{*} \text { in } M_{p}
$$

Thus, the Formula (21) holds by (20), for all $n \in \mathbb{N}$, for $k \in \mathbb{Z}$. 


\section{Semigroup $C^{*}$-Subalgebra $\mathfrak{S}_{p}$ of $M_{p}$}

Let $M_{p}$ be the $p$-adic $C^{*}$-algebra for $p \in \mathcal{P}$. Take projections

$$
P_{p, j}=\alpha_{\partial_{j}}^{p} \in M_{p}
$$

induced by boundaries $\partial_{j}$ of $\mathbb{Q}_{p}$, for all $j \in \mathbb{Z}$.

Definition 4. Fix $p \in \mathcal{P}$. Let $\mathfrak{S}_{p}$ be the $C^{*}$-subalgebra

$$
\mathfrak{S}_{p}=C^{*}\left(\left\{P_{p, j}\right\}_{j \in \mathbb{Z}}\right)=\overline{\mathbb{C}\left[\left\{P_{p, j}\right\}_{j \in \mathbb{Z}}\right]} \text { of } M_{p},
$$

where $P_{p, j}$ are projections (22), for all $j \in \mathbb{Z}$. We call this $C^{*}$-subalgebra $\mathfrak{S}_{p}$, the $p$-adic boundary $\left(C^{*}-\right)$ subalgebra of $M_{p}$.

Every $p$-adic boundary subalgebra $\mathfrak{S}_{p}$ satisfies the following structure theorem.

Proposition 5. Let $\mathfrak{S}_{p}$ be the $p$-adic boundary subalgebra (23) of $M_{p}$. Then,

$$
\mathfrak{S}_{p} \stackrel{*-i s o}{=} \underset{j \in \mathbb{Z}}{\oplus}\left(\mathbb{C} \cdot P_{p, j}\right) \stackrel{*-i s o}{=} \mathbb{C}^{\oplus \mathbb{Z}}
$$

in $M_{p}$.

Proof. It suffices to show that the generating projections $\left\{P_{p, j}\right\}_{j \in \mathbb{Z}}$ of $\mathfrak{S}_{p}$ are mutually orthogonal from each other. For any $j_{1}, j_{2} \in \mathbb{Z}$,

$$
P_{p, j_{1}} P_{p, j_{2}}=\alpha^{p}\left(\chi_{\partial_{j_{1}}^{p} \cap \partial_{j_{2}}^{p}}\right)=\delta_{j_{1}, j_{2}} \alpha_{\partial_{j_{1}}^{p}}^{p}=\delta_{j_{1}, j_{2}} P_{p, j_{1}} \text {, }
$$

in $\mathfrak{S}_{p}$. Therefore, the structure theorem (24) holds.

\section{Statistical Data Determined by $\left\{\boldsymbol{P}_{p, j}\right\}_{p \in \mathcal{P}, j \in \mathbb{Z}}$}

Let $\left(M_{p}, \varphi_{j}^{p}\right)$ be the $j$-th $p$-measure space for $j \in \mathbb{Z}$, and let $\mathfrak{S}_{p}$ be the boundary subalgebra (23) of $M_{p}$, satisfying the structure theorem (24). Throughout this section, fix a prime $p$. On the pair $\left(\mathfrak{S}_{p}, \varphi_{j}^{p}=\left.\varphi_{j}^{p}\right|_{\mathfrak{S}_{p}}\right)$, which is a sub-structure of $\left(M_{p}, \varphi_{j}^{p}\right)$, we have

$$
\varphi_{j}^{p}\left(P_{p, k}\right)=\delta_{j, k}\left(\frac{1}{p^{j}}-\frac{1}{p^{j+1}}\right), \forall j, k \in \mathbb{Z},
$$

by (21), where $P_{p, k}$ are the projections (22), generating $\mathfrak{S}_{p}$.

Now, let $\phi$ be the Euler totient function, which is an arithmetic function

$$
\phi: \mathbb{N} \rightarrow \mathbb{C},
$$

defined by

$$
\phi(n)=|\{k \in \mathbb{N}: k \leq n, \operatorname{gcd}(n, k)=1\}|,
$$

for all $n \in \mathbb{N}$, where $|X|$ mean the cardinalities of sets $X$, and gcd is the greatest common divisor.

By (26), one has

$$
\phi(q)=q-1=q\left(1-\frac{1}{q}\right), \forall q \in \mathcal{P} .
$$

Thus, one can get that 


$$
\varphi_{j}^{p}\left(P_{p, j}\right)=\frac{\phi(p)}{p^{j+1}}
$$

by (25) and (27), for $j \in \mathbb{Z}$.

Motivated by (28), define new linear functionals $\tau_{j}^{p}: \mathfrak{S}_{p} \rightarrow \mathbb{C}$, by linear morphisms,

$$
\tau_{j}^{p}=\frac{1}{\phi(p)} \varphi_{j}^{p} \text { on } \mathfrak{S}_{p}
$$

inducing new measure-theoretic structure,

$$
\mathfrak{S}_{p}(j) \stackrel{\text { denote }}{=}\left(\mathfrak{S}_{p}, \tau_{j}^{p}\right) \text {, for all } j \in \mathbb{Z},
$$

where $\tau_{j}^{p}$ are in the sense of (29).

Proposition 6. Let $\mathfrak{S}_{p}(j)=\left(\mathfrak{S}_{p}, \tau_{j}^{p}\right)$ be a pair (30), and let $P_{p, k}$ be the generating projections (22) of $\mathfrak{S}_{p}$, for all $k \in \mathbb{Z}$. Then,

$$
\tau_{j}^{p}\left(P_{p, k}^{n}\right)=\frac{\delta_{j, k}}{p^{j+1}}, \text { for all } n \in \mathbb{N} .
$$

Proof. The Formula (31) is proven by (28) and (29).

\subsection{Weighted-Semicircular Elements}

Let $(A, \varphi)$ be an arbitrary topological $*$-probability space ( $C^{*}$-probability space, or $W^{*}$-probability space, or Banach $*$-probability space, etc.), consisting of a topological $*$-algebra $A\left(C^{*}\right.$-algebra, resp., $W^{*}$-algebra, resp., Banach $*$-algebra), and a linear functional $\varphi$ on $A$. As usual in operator theory, an operator $a \in(A$, $\varphi$ ) is said to be self-adjoint, if $a=a^{*}$ in $A$, where $a^{*}$ is the adjoint of $a$.

Definition 5. A self-adjoint operator $a \in(A, \varphi)$ is said to be semicircular in $(A, \varphi)$, if

$$
\varphi\left(a^{n}\right)=\omega_{n} c_{\frac{n}{2}}, \text { for all } n \in \mathbb{N},
$$

with

$$
\omega_{n}= \begin{cases}1, & \text { if } n \text { is even, } \\ 0, & \text { if } n \text { is odd, }\end{cases}
$$

for all $n \in \mathbb{N}$, where $c_{k}$ are the $k$-th Catalan numbers,

$$
c_{k}=\frac{1}{k+1}\left(\begin{array}{c}
2 k \\
k
\end{array}\right)=\frac{1}{k+1} \frac{(2 k) !}{(k !)^{2}}=\frac{(2 k) !}{k !(k+1) !},
$$

for all $k \in \mathbb{N}_{0} \stackrel{\text { def }}{=} \mathbb{N} \cup\{0\}$.

It is well-known that, if $k_{n}(\ldots)$ is the free cumulant on $A$ in terms of $\varphi$ (in the sense of [11-13]), then a self-adjoint operator $a$ is semicircular in $(A, \varphi)$, if and only if

$$
k_{n}(\underbrace{a, a, \ldots . ., a}_{n \text {-times }})= \begin{cases}1, & \text { if } n=2, \\ 0, & \text { otherwise, }\end{cases}
$$

for all $n \in \mathbb{N}$ (e.g., [11,13]). The above characterization (33) of the semicircularity (32) is obtained by the Möbius inversion of [12]. Thus, we use the semicircularity (32) and its characterization (33) alternatively.

Motivated by (33), we define the following generalized concept of the semicircularity (32). 
Definition 6. Let $a \in(A, \varphi)$ be a self-adjoint operator. It is said to be weighted-semicircular in $(A, \varphi)$ with its weight $t_{0}$ (in short, $t_{0}$-semicircular), if there exists $t_{0} \in \mathbb{C}^{\times}=\mathbb{C} \backslash\{0\}$, such that

$$
k_{n}(\underbrace{a, a, \ldots, a}_{n \text {-times }})= \begin{cases}t_{0}, & \text { if } n=2, \\ 0, & \text { otherwise, }\end{cases}
$$

for all $n \in \mathbb{N}$, where $k_{n}(\ldots)$ is the free cumulant on $A$ in terms of $\varphi$.

By definition (34), and by the Möbius inversion of [12], one obtains the following free-moment characterization of (34): A self-adjoint operator $a$ is $t_{0}$-semicircular in $(A, \varphi)$, if and only if there exists $t_{0} \in$ $\mathbb{C}^{\times}$, such that

$$
\varphi\left(a^{n}\right)=\omega_{n} t_{0}^{\frac{n}{2}} c_{\frac{n}{2}}
$$

where $\omega_{n}$ and $c_{\frac{n}{2}}$ are in the sense of (32), for all $n \in \mathbb{N}$. (see [8] for details).

7.2. Tensor Product Banach $*$-Algebra $\mathfrak{L S}_{p}$

Let $\mathfrak{S}_{p}(k)=\left(\mathfrak{S}_{p}, \tau_{k}^{p}\right)$ be a pair (30) for $p \in \mathcal{P}, k \in \mathbb{Z}$. Define now bounded linear transformations $\mathbf{c}_{p}$ and $\mathbf{a}_{p}$ "acting on the $C^{*}$-algebra $\mathfrak{S}_{p}$," by linear morphisms satisfying,

$$
\begin{aligned}
& \mathbf{c}_{p}\left(P_{p, j}\right)=P_{p, j+1}, \\
& \text { and } \\
& \mathbf{a}_{p}\left(P_{p, j}\right)=P_{p, j-1},
\end{aligned}
$$

on $\mathfrak{S}_{p}$, for all $j \in \mathbb{Z}$. They are well-defined on $\mathfrak{S}_{p}$ by (24).

By (36), one can understand $\mathbf{c}_{p}$ and $\mathbf{a}_{p}$ as Banach-space operators contained in the operator space $B\left(\mathfrak{S}_{p}\right)$, consisting of all bounded linear operators acting on $\mathfrak{S}_{p}$, by regarding $\mathfrak{S}_{p}$ as a Banach space equipped with its $C^{*}$-norm (e.g., [2]).

Definition 7. The Banach-space operators $\mathbf{c}_{p}$ and $\mathbf{a}_{p}$ of (36) are called the $p$-creation, respectively, the p-annihilation on $\mathfrak{S}_{p}$. Define a new Banach-space operator $\mathbf{1}_{p}$ by

$$
\mathbf{1}_{p}=\mathbf{c}_{p}+\mathbf{a}_{p} \text { on } \mathfrak{S}_{p} .
$$

We call this operator $\mathbf{1}_{p}$ of (37), the p-radial operator on $\mathfrak{S}_{p}$.

Let $\mathbf{1}_{p}$ be the $p$-radial operator (37) in $B\left(\mathfrak{S}_{p}\right)$. Construct a closed subspace $\mathfrak{L}_{p}$ of $B\left(\mathfrak{S}_{p}\right)$ by

$$
\mathfrak{L}_{p}=\overline{\mathbb{C}}\left[\left\{\mathbf{1}_{p}\right\}\right] \text { in } B\left(\mathfrak{S}_{p}\right),
$$

where $\bar{Y}$ are the operator-norm closures of subsets $Y$ of the operator space $B\left(\mathfrak{S}_{p}\right)$.

By definition (38), $\mathfrak{L}_{p}$ is not only a subspace of $B\left(\mathfrak{S}_{p}\right)$, but also an algebra. In addition, hence, it is a well-defined Banach algebra. On this Banach algebra $\mathfrak{L}_{p}$, define a unary operation $(*)$ by

$$
\sum_{k=0}^{\infty} s_{k} \mathbf{l}_{p}^{k} \in \mathfrak{L}_{p} \longmapsto \sum_{k=0}^{\infty} \overline{s_{k}} \mathbf{l}_{p}^{k} \in \mathfrak{L}_{p}
$$

with axiomatization:

$$
\mathbf{1}_{p}^{0}=1_{\mathfrak{S}_{p}}, \text { the identity operator of } B\left(\mathfrak{S}_{p}\right)
$$

satisfying 


$$
1_{\mathfrak{S}_{p}}(Y)=Y, \text { for all } Y \in \mathfrak{S}_{p},
$$

where $s_{k} \in \mathbb{C}$, with their conjugates $\overline{s_{k}} \in \mathbb{C}$.

Then, the operation (39) is a well-defined adjoint on $\mathfrak{L}_{p}$ (e.g., $\left.[2,8,10]\right)$. Thus, equipped with the adjoint (39), this Banach algebra $\mathfrak{L}_{p}$ of (38) forms a Banach $*$-algebra embedded in $B\left(\mathfrak{S}_{p}\right)$.

Let $\mathfrak{L}_{p}$ be the above Banach $*$-algebra acting on $\mathfrak{S}_{p}$. Construct now the tensor product Banach $*$-algebra $\mathfrak{L} \mathfrak{S}_{p}$ by

$$
\mathfrak{L S}_{p}=\mathfrak{L}_{p} \otimes_{\mathbb{C}} \mathfrak{S}_{p},
$$

where $\otimes_{\mathbb{C}}$ is the tensor product of Banach $*$-algebras.

Note that operators $\mathbf{1}_{p}^{n} \otimes P_{p, j}$ generate $\mathfrak{L S}_{p}$, for all $n \in \mathbb{N}_{0}$, and $j \in \mathbb{Z}$, where $P_{p, j}$ are the projections (22) of $\mathfrak{S}_{p}$, by the definition (38) of the tensor factor $\mathfrak{L}_{p}$ of $\mathfrak{L S}_{p}$, and the structure theorem (24) of the other tensor factor $\mathfrak{S}_{p}$ of $\mathfrak{L S}_{p}$.

Define a linear morphism $E_{p}: \mathfrak{L S}_{p} \rightarrow \mathfrak{S}_{p}$ by a linear transformation satisfying that:

$$
E_{p}\left(l_{p}^{k} \otimes P_{p, j}\right)=\frac{\left(p^{j+1}\right)^{k+1}}{\left[\frac{k}{2}\right]+1} l_{p}^{k}\left(P_{p, j}\right)
$$

for all $k \in \mathbb{N}_{0}, j \in \mathbb{Z}$, where $\left[\frac{k}{2}\right]$ is the minimal integer greater than or equal to $\frac{k}{2}$, for all $k \in \mathbb{N}_{0}$. By (24), (38) and (40), this morphism $E_{p}$ of (41) is indeed a well-defined linear transformation.

Now, consider how our $p$-radial operator $\mathbf{l}_{p}=\mathbf{c}_{p}+\mathbf{a}_{p}$ acts on $\mathfrak{S}_{p}$. First, observe that

$$
\mathbf{c}_{p} \mathbf{a}_{p}\left(P_{p, j}\right)=P_{p, j}=\mathbf{a}_{p} \mathbf{c}_{p}\left(P_{p, j}\right),
$$

for all $j \in \mathbb{Z}, p \in \mathcal{P}$. Thus, one has

$$
\mathbf{c}_{p}^{n_{1}} \mathbf{a}_{p}^{n_{2}}=\mathbf{a}_{p}^{n_{2}} \mathbf{c}_{p}^{n_{1}}, \text { on } \mathfrak{S}_{p}
$$

by (42), for all $n, n_{1}, n_{2} \in \mathbb{N}$.

Thus, we have

$$
\mathbf{1}_{p}^{n}=\left(\mathbf{c}_{p}+\mathbf{a}_{p}\right)^{n}=\sum_{k=0}^{n}\left(\begin{array}{c}
n \\
k
\end{array}\right) \mathbf{c}_{p}^{k} \mathbf{a}_{p}^{n-k} \text { on } \mathfrak{S}_{p}
$$

with identity:

$$
\mathbf{c}_{p}^{0}=1_{\mathfrak{S}_{p}}=\mathbf{a}_{p}^{0},
$$

for all $n \in \mathbb{N}$, by (43), where

$$
\left(\begin{array}{l}
n \\
k
\end{array}\right)=\frac{n !}{k !(n-k) !}, \text { for all } k \leq n \in \mathbb{N}_{0} .
$$

Proposition 7. Let $\mathbf{1}_{p} \in \mathfrak{L}_{p}$ be the p-radial operator on $\mathfrak{S}_{p}$. Then,

(i) $\mathbf{1}_{p}^{2 m-1}$ does not contain $1_{\mathfrak{S}_{p}}$-term, and

(ii) $\mathbf{1}_{p}^{2 m}$ contains its $1_{\mathfrak{S}_{p}}$-term, $\left(\begin{array}{c}2 m \\ m\end{array}\right) \cdot 1_{\mathfrak{S}_{p}}$, for all $m \in \mathbb{N}$.

Proof. The proofs of (i) and (ii) in Proposition 7 are done by straightforward computations of (44) under (43). See [8] for details. 


\subsection{Generating Operators $\left\{Q_{p, j}\right\}_{j \in \mathbb{Z}}$ of $\mathfrak{L S}_{p}$}

Fix $p \in \mathcal{P}$, and let $\mathfrak{L S}_{p}$ be the tensor product Banach $*$-algebra (40), and let $E_{p}: \mathfrak{L S}_{p} \rightarrow \mathfrak{S}_{p}$ be the linear transformation (41). Throughout this section, let

$$
Q_{p, j}=\mathbf{l}_{p} \otimes P_{p, j} \in \mathfrak{L S}_{p},
$$

for $j \in \mathbb{Z}$, where $P_{p, j}$ are projections (22) generating $\mathfrak{S}_{p}$. Observe that

$$
\begin{aligned}
Q_{p, j}^{n} & =\left(\mathbf{1}_{p} \otimes P_{p, j}\right)^{n} \\
& =\mathbf{l}_{p}^{n} \otimes P_{p, j}^{n}=\mathbf{1}_{p}^{n} \otimes P_{p, j},
\end{aligned}
$$

for all $n \in \mathbb{N}$, for all $j \in \mathbb{Z}$. Thus, these operators $\left\{Q_{p, j}\right\}_{j \in \mathbb{Z}}$ of (45) generate the Banach $*$-algebra $\mathfrak{L S}_{p}$, by (40).

Consider now that, if $Q_{p, j} \in \mathfrak{L} \mathfrak{S}_{p}$ is in the sense of (45) for $j \in \mathbb{Z}$, then

$$
E_{p}\left(Q_{p, j}^{n}\right)=\frac{\left(p^{j+1}\right)^{n+1}}{\left[\frac{n}{2}\right]+1} \mathbf{1}_{p}^{n}\left(P_{p, j}\right)
$$

by (41) and (46), for all $n \in \mathbb{N}$.

For any fixed $j \in \mathbb{Z}$, define a linear functional $\tau_{p, j}^{0}$ on $\mathfrak{L S}_{p}$ by

$$
\tau_{p, j}^{0}=\tau_{j}^{p} \circ E_{p} \text { on } \mathfrak{L S}_{p}
$$

where $\tau_{j}^{p}$ is a linear functional (29) on $\mathfrak{S}_{p}$. The pair $\left(\mathfrak{L} \mathfrak{S}_{p}, \tau_{p, j}^{0}\right)$ forms a new measure- theoretic structure.

By (47) and (48),

$$
\tau_{p, j}^{0}\left(Q_{p, j}^{n}\right)=\frac{\left(p^{j+1}\right)^{n+1}}{\left[\frac{n}{2}\right]+1} \tau_{j}^{p}\left(\mathbf{l}_{p}^{n}\left(P_{p, j}\right)\right)
$$

for all $n \in \mathbb{N}$.

Lemma 1. Fix $j \in \mathbb{Z}$, and the pair $\left(\mathfrak{L} \mathfrak{S}_{p}, \tau_{p, j}^{0}\right)$. Let $Q_{p, k}=\mathbf{1}_{p} \otimes P_{p, k} \in\left(\mathfrak{L} \mathfrak{S}_{p}, \tau_{p, j}^{0}\right)$, for all $k \in \mathbb{Z}$. Then,

$$
\tau_{p, j}^{0}\left(Q_{p, k}^{n}\right)=\delta_{j, k}\left(\omega_{n}\left(p^{2(j+1)}\right)^{\frac{n}{2}} c_{\frac{n}{2}}\right)
$$

for all $n \in \mathbb{N}$, where $\omega_{n}$ are in the sense of (32).

Proof. Suppose $k=j$ in $\mathbb{Z}$. Then, the formula (50) is proven by (49) with help of (i) in Proposition 7 and (ii) in Proposition 7. Meanwhile, if $k \neq j$ in $\mathbb{Z}$, then

$$
\tau_{p, j}^{0}\left(Q_{p, k}^{n}\right)=0
$$

by (29) and (47). Therefore, the above statistical data (50) holds. See [8] and [10] for details.

\section{Weighted-Semicircularity on $\mathfrak{L} \mathfrak{S}$}

Let $\mathfrak{L S}_{p}$ and $\tau_{p, j}^{0}$ be in the sense of (40), respectively, (48). Then, one has the corresponding measure-theoretic pairs,

$$
\mathfrak{L S}_{p}(j)=\left(\mathfrak{L} \mathfrak{S}_{p}, \tau_{p, j}^{0}\right)
$$

for all $p \in \mathcal{P}, j \in \mathbb{Z}$.

Let $Q_{p, k}=\mathbf{1}_{p} \otimes P_{p, k}$ be the generating elements (45) of $\mathfrak{L S}_{p}(j)$, for $p \in \mathcal{P}, k \in \mathbb{Z}$. Then, by (50), one has

$$
\tau_{p, j}^{0}\left(Q_{p, j}^{n}\right)=\omega_{n}\left(p^{2(j+1)}\right)^{\frac{n}{2}} c_{\frac{n}{2}}
$$

for all $p \in \mathcal{P}, j \in \mathbb{Z}$, for all $n \in \mathbb{N}$, where $\omega_{n}$ are in the sense of (32). 


\subsection{Free Product Banach $*$-Probability Space $\left(\mathfrak{L S}, \tau^{0}\right)$}

Construct the family

$$
\left\{\mathfrak{L} \mathfrak{S}_{p}(j)=\left(\mathfrak{L} \mathfrak{S}_{p}, \tau_{p, j}^{0}\right): p \in \mathcal{P}, j \in \mathbb{Z}\right\}
$$

of the measure-theoretic structures (51), and define the free product Banach *-probability space,

$$
\begin{aligned}
& \left(\mathfrak{L S}, \tau^{0}\right) \stackrel{\text { def }}{=} \underset{p \in \mathcal{P}, j \in \mathbb{Z}}{\star} \mathfrak{L S}_{p}(j),
\end{aligned}
$$

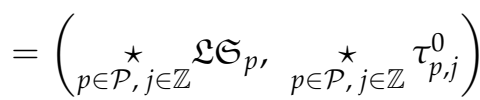

in the sense of [15] (e.g., [11-14]). For example, the structures $\mathfrak{L S}_{p}(j)$ of (51) are the free blocks of this Banach $*$-probability space $\left(\mathfrak{L} \mathfrak{S}, \tau^{0}\right)$ of $(53)$.

Definition 8. The Banach $*$-probability space

$$
\mathfrak{L S} \stackrel{\text { denote }}{=}\left(\mathfrak{L} \mathfrak{S}, \tau^{0}\right) \text { of }(53)
$$

is called the free Adelic filterization.

Let $\mathfrak{L} \mathfrak{S}$ be the free Adelic filterization (53). Take a subset $\mathcal{Q}$,

$$
\mathcal{Q}=\left\{Q_{p, j} \in \mathfrak{L S}_{p}(j)\right\}_{p \in \mathcal{P}, j \in \mathbb{Z}}
$$

of $\mathfrak{L S}$.

Theorem 1. The operators $Q_{p, j}$ of the family $\mathcal{Q}$ of $(54)$ are $p^{2(j+1)}$-semicircular in the free Adelic filterization $\mathfrak{L} \mathfrak{S}_{\text {, }}$ for all $p \in \mathcal{P}, j \in \mathbb{Z}$. More precisely,

$$
\tau^{0}\left(Q_{p, j}^{n}\right)=\omega_{n} p^{n(j+1)} c_{\frac{n}{2}}
$$

and

$$
k_{n}^{0}(\underbrace{Q_{p, j}, Q_{p, j}, \ldots, Q_{p, j}}_{n \text {-times }})= \begin{cases}p^{2(j+1)}, & \text { if } n=2, \\ 0, & \text { otherwise, }\end{cases}
$$

for all $n \in \mathbb{N}$, where $k_{n}^{0}(\ldots)$ is the free cumulant on $\mathfrak{L} \mathfrak{S}$ in terms of $\tau^{0}$.

Proof. Observe first that the operators $Q_{p, j} \in \mathcal{Q}$ are self-adjoint in $\mathfrak{L} \mathfrak{S}$, since

$$
Q_{p, j}^{*}=\mathbf{1}_{p}^{*} \otimes P_{p, j}^{*}=\mathbf{1}_{p} \otimes P_{p, j}=Q_{p, j} \text { in } \mathfrak{L} \mathfrak{S} .
$$

By (54), every operator $Q_{p, j} \in \mathcal{Q}$ is taken from a free block $\mathfrak{L S}_{p}(j)$, and, hence, $Q_{p, j}^{n}$ are contained in the same block $\mathfrak{L S}_{p}(j)$, as free reduced words of $\mathfrak{L} \mathfrak{S}$ with their length-1, for all $n \in \mathbb{N}$, for all $p \in \mathcal{P}, j \in \mathbb{Z}$. Therefore, by (53),

$$
\tau^{0}\left(Q_{p, j}^{n}\right)=\tau_{p, j}^{0}\left(Q_{p, j}^{n}\right)=\omega_{n} p^{n(j+1)} c_{\frac{n}{2}}
$$

for all $n \in \mathbb{N}$, by (52).

In addition, by the Möbius inversion of [12], one has 


$$
\begin{aligned}
k_{n}^{0}\left(Q_{p, j}, \ldots, Q_{p, j}\right) & =k_{n}^{0, p, j}\left(Q_{p, j}, \ldots, Q_{p, j}\right) \\
& = \begin{cases}p^{2(j+1)}, & \text { if } n=2, \\
0, & \text { otherwise }\end{cases}
\end{aligned}
$$

for all $n \in \mathbb{N}$, where $k_{n}^{0, p, j}(\ldots)$ is the free cumulant on $\mathfrak{L S}_{p}(j)$ in terms of $\tau_{p, j}^{0}$, for all $p \in \mathcal{P}, j \in \mathbb{Z}$. Therefore, by (34) and (35), these self-adjoint operators $Q_{p, j} \in \mathcal{Q}$ are $p^{2(j+1)}$-semicircular in $\mathfrak{L} \mathfrak{S}$.

By the above weighted-semicircularity on $\mathfrak{L} \mathfrak{S}$, one obtains the following semicircularity on $\mathfrak{L} \mathfrak{S}$.

Theorem 2. Let $Q_{p, j} \in \mathcal{Q}$ in the free Adelic filterization $\mathfrak{L} \mathfrak{S}$ of (53), where $\mathcal{Q}$ is the family (54), for $p \in \mathcal{P}, j \in \mathbb{Z}$. Then, the operators

$$
\Theta_{p, j}=\frac{1}{p^{j+1}} Q_{p, j} \in \mathfrak{L} \mathfrak{S}
$$

are semicircular in $\mathfrak{L} \mathfrak{S}$, satisfying

$$
\begin{aligned}
& \tau^{0}\left(\Theta_{p, j}^{n}\right)=\omega_{n} c_{\frac{n}{2}}, \\
& \text { and } \\
& k_{n}^{0}(\underbrace{\Theta_{p, j}, \Theta_{p, j}, \ldots, \Theta_{p, j}}_{n \text {-times }})=\left\{\begin{array}{l}
1, \text { if } n=2, \\
0, \text { otherwise, }
\end{array}\right.
\end{aligned}
$$

for all $n \in \mathbb{N}$.

Proof. Let $\Theta_{p, j}=\frac{1}{p^{j+1}} Q_{p, j}$ be in the sense of (55), where $Q_{p, j} \in \mathcal{Q}$ are the $p^{2(j+1)}$-semicircular elements of $\mathfrak{L S}$, for $p \in \mathcal{P}, j \in \mathbb{Z}$. By the self-adjointness of $Q_{p, j}$, the operator $\Theta_{p, j}$ is self-adjoint in $\mathfrak{L} \mathfrak{S}$, too, because $\frac{1}{p^{j+1}} \in \mathbb{R}^{\times}$in $\mathbb{C}$, for all $\mathcal{P}, j \in \mathbb{Z}$.

Since such a self-adjoint operator $\Theta_{p, j} \in \mathcal{X}$ is contained in the free block $\mathfrak{L S}_{p}(j)$ of $\mathfrak{L} \mathfrak{S}$, the operators $\Theta_{p, j}^{n}$ are contained in the same free block $\mathfrak{L S}_{p}(j)$ in $\mathfrak{L} \mathfrak{S}$, for all $n \in \mathbb{N}$, as free reduced words with their lengths-1. Thus, one has that

$$
\begin{aligned}
\tau^{0}\left(\Theta_{p, j}^{n}\right) & =\tau_{p, j}^{0}\left(\Theta_{p, j}^{n}\right)=\tau_{p, j}^{0}\left(\frac{1}{p^{n(j+1)}} Q_{p, j}^{n}\right) \\
& =\left(\frac{1}{p^{j+1}}\right)^{n} \tau_{p, j}^{0}\left(Q_{p, j}^{n}\right)=\left(\frac{1}{p^{j+1}}\right)^{n}\left(\omega_{n} p^{n(j+1)} c_{\frac{n}{2}}\right)
\end{aligned}
$$

by the $p^{2(j+1)}$-semicircularity of $Q_{p, j} \in \mathcal{Q}$ in $\mathfrak{L} \mathfrak{S}$

$$
=\omega_{n} c_{\frac{n}{2}}
$$

for all $n \in \mathbb{N}$. Therefore, by (32) and (33), the operator $\Theta_{p, j}$ is semicircular in $\mathfrak{L} \mathfrak{S}$.

In addition, by (34) and (57), one obtains the free cumulant formula in (56) by the Möbius inversion of [12].

Let

$$
\begin{aligned}
\mathcal{X} & =\left\{\Theta_{p, j} \in \mathfrak{L S}_{p}(j) \mid p \in \mathcal{P}, j \in \mathbb{Z}\right\} \\
& =\left\{\Theta_{p, j}=\frac{1}{p^{j+1}} Q_{p, j} \in \mathfrak{L} \mathfrak{S} \mid Q_{p, j} \in \mathcal{Q}\right\}
\end{aligned}
$$


be a subset of $\mathfrak{L} \mathfrak{S}$ consisting of the operators $\Theta_{p, j}$ of (55) induced by the family $\mathcal{Q}$ of (54).

Recall that a subset $S$ of an arbitrary $*$-probability space $(A, \varphi)$ is said to be a free family, if all elements of $S$ are mutually free from each other in $(A, \varphi)$ (e.g., [11,15]).

Definition 9. A free family $S$ is said to be a free (weighted-)semicircular family, if every element of $S$ is (weighted)semicircular in a topological $*$-probability space $(A, \varphi)$.

Thus, by (53), (54) and (58), we obtain the following result.

Corollary 1. Let $\mathfrak{L} \mathfrak{S}$ be the free Adelic filterization (53).

The family $\mathcal{Q}$ of (54) is a free weighted-semicircular family in $\mathfrak{L} \mathfrak{S}$.

The family $\mathcal{X}$ of $(58)$ is a free semicircular family in $\mathfrak{L} \mathfrak{S}$.

Proof. The proofs of the statements (59) and (60) are done by (54), respectively, by (58). See [8] for details.

\subsection{Free-Semicircular Adelic Filterization $\mathbb{L} \mathbb{S}$}

Let $\mathfrak{L} \mathfrak{S}$ be the free Adelic filterization (53), and let $\mathcal{Q}$ be the free weighted-semicircular family (59), and $\mathcal{X}$, the free semicircular family (60) in $\mathfrak{L} \mathfrak{S}$. We now focus on the Banach $*$-subalgebra $\mathbb{L} \mathfrak{S}$ of $\mathfrak{L} \mathfrak{S}$ generated by the free family $\mathcal{X}$,

$$
\mathbb{L} \mathbb{S} \stackrel{\operatorname{def}}{=} \overline{\mathbb{C}[\mathcal{X}]} \subset \mathfrak{L} \mathfrak{S},
$$

where $\bar{X}$ are the Banach-topology closures of subsets $X$ of $\mathfrak{L} \mathfrak{S}$.

By (61), we obtain the corresponding Banach $*$-probability space,

$$
\mathbb{L} \mathbb{S} \stackrel{\text { denote }}{=}\left(\mathbb{L} \mathbb{S}, \tau^{0}=\left.\tau^{0}\right|_{\mathbb{L S}}\right)
$$

as a free-probabilistic sub-structure of $\mathfrak{L} \mathfrak{S}$.

Definition 10. Let $\mathbb{L} \mathbb{S}=\left(\mathbb{L} S, \tau^{0}\right)$ be the Banach $*$-probability space (62) in the free Adelic filterization $\mathfrak{L} \mathfrak{S}$ of (53). Then, it is called the (free-)semicircular Adelic filterization (of $\mathfrak{L S}$, generated by the free semicircular family $\mathcal{X}$ of (60)).

Let $\mathbb{L} \mathbb{S}$ be the semicircular Adelic filterization (62). Then, it satisfies the following structure theorem in $\mathfrak{L S}$.

Theorem 3. Let $\mathbb{L} \mathbb{S}$ be the semicircular Adelic filterization (62) of the free Adelic filterization $\mathfrak{L} S$. Then, the Banach *-algebra $\mathbb{L} \mathbb{S}$ satisfies that

$$
\begin{aligned}
& \mathbb{L S} \stackrel{* \text {-iso }}{=} \underset{p \in \mathcal{P}, j \in \mathbb{Z}}{\star}\left(\overline{\mathbb{C}\left[\left\{\Theta_{p, j}\right\}\right]}\right) \\
& \stackrel{*-i s o}{=} \overline{\mathbb{C}\left[{ }_{p \in \mathcal{P}, j \in \mathbb{Z}}^{\star}\left\{\Theta_{p, j}\right\}\right]}
\end{aligned}
$$

in $\mathfrak{L S}$, where the free product $(\star)$ in the first isomorphic relation of (63) means the free-probability-theoretic free product of $[11,15]$ (with respect to the linear functional $\tau^{0}$ of (62)), and the free product $(\star)$ in the second isomorphic relation of (63) is the pure-algebraic free product inducing noncommutative free words in $\mathcal{X}$. 
Proof. By the definition (62) of our semicircular Adelic filterization $\mathbb{L} \mathbb{S}$, we have

$$
\begin{aligned}
& \mathbb{L} \mathbb{S}=\overline{\mathbb{C}[\mathcal{X}]}=\overline{\mathbb{C}\left[\left\{\Theta_{p, j} \in \mathcal{X}: p \in \mathcal{P}, j \in \mathbb{Z}\right\}\right]}
\end{aligned}
$$

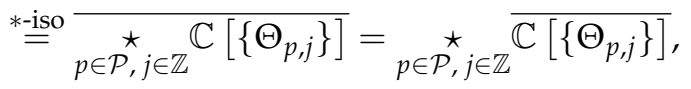

since $\mathcal{X}$ is a free family in $\mathfrak{L} \mathfrak{S}$, by $(60)$.

By (64), all operators in $\mathbb{L} \mathbb{S}$ are limits of linear combinations of free reduced words in $\mathcal{X}$ (e.g., [11-15]). By the self-adjointness of all free generators $\Theta_{p, j}$ in $\mathcal{X}$, every (algebraic) free word in $\mathcal{X}$ has its unique operator-form in $\mathbb{L} \mathbb{S}$, as a free "reduced" word. Therefore,

$$
\begin{aligned}
& \underset{p \in \mathcal{P}, j \in \mathbb{Z}}{\star} \overline{\mathbb{C}\left[\left\{\Theta_{p, j}\right\}\right]} \stackrel{\text {-iso }}{=} \overline{\mathbb{C}[\{\text { free reduced words in } \mathcal{X}\}]} \\
& =\overline{\mathbb{C}[\{\text { free words in } \mathcal{X}\}]} \\
& =\overline{\mathbb{C}\left[{ }_{p \in \mathcal{P}, j \in \mathbb{Z}}^{\star}\left\{\Theta_{p, j}\right\}\right]},
\end{aligned}
$$

i.e., by (64) and (65), the second $*$-isomorphic relation of (63) holds true (e.g., [23]).

In addition, one obtains the following set-identity.

Corollary 2. Let $\mathbb{L} \mathbb{S}$ be the semicircular Adelic filterization (61). Then,

$$
\mathbb{L} \mathbb{S}=\overline{\mathbb{C}[\mathcal{Q}]} \text { in } \mathfrak{L S},
$$

where $\mathcal{Q}$ is the free weighted-semicircular family (59) of $\mathfrak{L} \mathfrak{S}$.

Proof. Observe that

$$
\begin{aligned}
\mathbb{L} \stackrel{\text { def }}{=} \overline{\mathbb{C}[\mathcal{X}]} & =\overline{\mathbb{C}\left[\left\{\Theta_{p, j} \in \mathfrak{L S}_{p}(j): p \in \mathcal{P}, j \in \mathbb{Z}\right\}\right]} \\
& =\overline{\mathbb{C}\left[\left\{p^{j+1} \Theta_{p, j}: \Theta_{p, j} \in \mathcal{X}\right\}\right]}=\overline{\mathbb{C}[\mathcal{Q}]}
\end{aligned}
$$

So, the set-equality (66) holds.

\section{Shifts on $\mathcal{P}$ Acting on $\mathbb{L} S$}

In this section, we study certain $*$-homomorphisms acting on $\mathbb{L} \mathbb{S}$ induced by shift processes on $\mathcal{P}$.

\subsection{Shifts on $\mathcal{P}$}

Let $\mathcal{P}$ be the set of all primes in $\mathbb{N}$. Since the set $\mathbb{N}$ of all natural numbers is a totally ordered set (or, TOset) under the usual inequality $(\leq)$, the subset $\mathcal{P}$ is a TOset under $(\leq)$ too. Without loss of generality, one can index $\mathcal{P}$ orderly by

$$
\mathcal{P}=\left\{q_{1} \leq q_{2} \leq q_{3} \leq q_{4} \leq \cdots\right\}
$$

with

$$
q_{1}=2, q_{2}=3, q_{3}=5, q_{4}=7, \ldots, \text { etc. }
$$

Define now a function $g: \mathcal{P} \rightarrow \mathcal{P}$ by

$$
g\left(q_{k}\right)=q_{k+1}, \text { for all } k \in \mathbb{N} .
$$


For the function $g$ of (68), we define $g^{n}: \mathcal{P} \rightarrow \mathcal{P}$ by

$$
\begin{aligned}
& g^{n}=\underbrace{g \circ g \circ g \circ \cdots \circ g}_{n \text {-times }}, \\
& \text { with axiomatization: } \\
& g^{0}=i d_{\mathcal{P}}, \text { the identity map on } \mathcal{P},
\end{aligned}
$$

for all $n \in \mathbb{N}_{0}$, where (o) is the usual functional composition.

By (69), one can have

$$
g^{n}\left(q_{k}\right)=q_{k+n} \text { in } \mathcal{P}, \text { for all } k \in \mathbb{N},
$$

for all $n \in \mathbb{N}_{0}$.

Definition 11. Let $g^{n}$ be in the sense of (69) for all $n \in \mathbb{N}_{0}$. Then, these functions $g^{n}$ on $\mathcal{P}$ are said to be $n$-shifts on $\mathcal{P}$, for all $n \in \mathbb{N}_{0}$. The 1-shift $g=g^{1}$ of (68) is simply called the shift on $\mathcal{P}$.

\subsection{Prime-Shift $*$-Homomorphisms on $\mathbb{L} \mathbb{S}$}

Let $\mathbb{L} \mathbb{S}$ be the semicircular Adelic filterization (63) generated by the free semicircular family $\mathcal{X}$ of (60), and let $g^{n}$ be the $n$-shifts (69) on the TOset $\mathcal{P}$ of (67), for $n \in \mathbb{N}_{0}$.

Define a bounded "multiplicative" linear transformation satisfying

$$
G\left(\Theta_{p, j}\right)=\Theta_{g(p), j}, \text { for all } \Theta_{p, j} \in \mathcal{X},
$$

for all $p \in \mathcal{P}, j \in \mathbb{Z}$, where $g=g^{1}$ is the shift on $\mathcal{P}$.

This multiplicative linear transformation $G$ of (70) is well-defined by (61) and (63), since all generators $\Theta_{p, j} \in \mathcal{X}$ of $\mathbb{L} \mathbb{S}$ are self-adjoint. Thus, if

$$
S=\prod_{l=1}^{N} \Theta_{p_{l}, j_{l},}^{n_{l}} \text { and } X=\prod_{l=1}^{N} Q_{p_{l}, j_{l}}^{n_{l}}
$$

are operator products of $\mathbb{L} \mathbb{S}$ in $\mathcal{X}$, respectively, in $\mathcal{Q}$, for $n_{1}, \ldots, n_{N} \in \mathbb{N}$, then

$$
\begin{aligned}
& G(S)=\prod_{l=1}^{N} \Theta_{g\left(p_{l}\right), j_{l}{ }^{\prime}}^{n_{l}} \\
& \text { and } \\
& G(X)=\left(\prod_{l=1}^{N} p_{l}^{n_{l}\left(j_{l}+1\right)}\right)\left(\prod_{l=1}^{N} \Theta_{g\left(p_{l}\right), j_{l}}^{n_{l}}\right),
\end{aligned}
$$

and, hence,

$$
G(X)=\left(\prod_{l=1}^{N} p_{l}^{n_{l}\left(j_{l}+1\right)}\right) G(S),
$$

in $\mathbb{L} \mathbb{S}$, by (71a). It is not difficult to check that

$$
\begin{aligned}
G\left(\left(t \Theta_{p, j}\right)^{*}\right) & =G\left(\bar{t} \Theta_{p, j}\right)=\bar{t} \Theta_{g(p), j} \\
& =\left(t \Theta_{g(p), j}\right)^{*}=\left(G\left(t \Theta_{p, j}\right)\right)^{*},
\end{aligned}
$$

for all $t \in \mathbb{C}$, and $\Theta_{p, j} \in \mathcal{X}$. It implies that

$$
G\left(T^{*}\right)=(G(T))^{*}, \text { for all } T \in \mathbb{L} \mathbb{S},
$$


by (63), (71a) and (71b). Thus, this bounded multiplicative linear transformation $G$ is adjoint-preserving, and, hence, it is a well-defined $*$-homomorphism on $\mathbb{L} \mathbb{S}$.

For the $*$-homomorphism $G$ of (70), one can have the iterated products (or compositions) $G^{n}$ of (n-copies of) $G$, as new $*$-homomorphisms on $\mathbb{L} \mathbb{S}$, with $G^{1}=G$, for all $n \in \mathbb{N}_{0}$, with identity

$$
G^{0}=1_{\mathbb{L} \mathbb{S}} \text {, the identity operator on } \mathbb{L} \mathbb{S},
$$

satisfying

$$
G^{0}\left(\Theta_{p, j}\right)=\Theta_{g^{0}(p), j}=\Theta_{p, j}=1_{\mathbb{L S}}\left(\Theta_{p, j}\right)
$$

for all $\Theta_{p, j} \in \mathcal{X}$.

More precisely, the morphisms $G^{n}$ satisfy

$$
G^{n}\left(\Theta_{p, j}\right)=\Theta_{g^{n}(p), j} \text { in } \mathbb{L} \mathbb{S}, \forall n \in \mathbb{N}_{0},
$$

for all $\Theta_{p, j} \in \mathcal{X}$, in $\mathbb{L} \mathbb{S}$, where $g^{n}$ are the $n$-shifts (69) on $\mathcal{P}$.

Definition 12. The *-homomorphism $G$ of (70) on the semicircular Adelic filterization $\mathbb{L} \mathbb{S}$ is called the prime-shift (*-homomorphism) on $\mathbb{L} \mathbb{S}$. In addition, the $n$-th powers $G^{n}$ of $(72)$ are called the n-prime-shift (*-homomorphism)s on $\mathbb{L} \mathbb{S}$, for all $n \in \mathbb{N}_{0}$.

By (72), we obtain the following result.

Theorem 4. Let $S=\Theta_{p, j}^{m}$, and $X=Q_{p, j}^{m}$ in $\mathbb{L} \mathbb{S}$, for $\Theta_{p, j} \in \mathcal{X}$, and $Q_{p, j} \in \mathcal{Q}$, for $m \in \mathbb{N}$. Then,

$$
\tau^{0}\left(\left(G^{n}(S)\right)^{k}\right)=\omega_{k m} c_{\frac{k m}{2}}=\tau^{0}\left(S^{k}\right)
$$

and

$$
\tau^{0}\left(\left(G^{n}(X)\right)^{k}\right)=\omega_{k m} p^{k m(j+1)} c_{\frac{k m}{2}}=\tau^{0}\left(X^{k}\right),
$$

for all $k \in \mathbb{N}$, where $G^{n}$ are the $n$-shift on $\mathcal{P}$, for all $n \in \mathbb{N}_{0}$.

Proof. Let $S$ and $X$ be given as above for a fixed $m \in \mathbb{N}$. Then, they are free reduced words with their lengths-1 in $\mathbb{L} \mathbb{S}$. Moreover,

$$
G^{n}(S)^{k}=\Theta_{q, j^{\prime}}^{k} \text { and } G^{n}(X)^{k}=p^{k(j+1)} \Theta_{q, j^{\prime}}^{k}
$$

by (71a), (71b) and (72), for all $k \in \mathbb{N}$, where

$$
q=g^{n}(p) \in \mathcal{P}, \text { for } n \in \mathbb{N}_{0}
$$

Therefore, one obtains the first free-distributional data in (73) by the semicircularity of $\Theta_{q, j} \in \mathcal{X}$. In addition, the second free-distributional data in (73) is obtained because

$$
\begin{aligned}
\tau^{0}\left(\left(G^{n}(X)\right)^{k}\right) & =p^{k(j+1)} \tau^{0}\left(\left(G^{n}\left(\Theta_{p, j}^{m}\right)\right)^{k}\right) \\
& =p^{k m(j+1)} \tau^{0}\left(S^{k}\right) \\
& =p^{k m(j+1)}\left(\omega_{k m} c_{\frac{k}{2}}\right)=\tau^{0}\left(X^{k}\right),
\end{aligned}
$$

by (50) and (71b) for all $k \in \mathbb{N}$, for all $n \in \mathbb{N}_{0}$.

Therefore, the formula (73) holds. 
The above free-distributional data (73) illustrate that the $n$-prime shifts $G^{n}$ of (72) preserve the free distributions of free reduced words of $\mathbb{L S}$ in $\mathcal{Q} \cup \mathcal{X}$ because the free distributions of free generators are preserved by $G^{n}$, for all $n \in \mathbb{N}_{0}$.

\subsection{Free-Homomorphisms on $\mathbb{L} \mathbb{S}$}

In this section, motivated by (73), we consider free-homomorphic relations on $\mathbb{L} \mathbb{S}$ under $n$-prime shifts $G^{n}$, for $n \in \mathbb{N}_{0}$.

Definition 13. Let $\left(A_{1}, \varphi_{1}\right)$, and $\left(A_{2}, \varphi_{2}\right)$ be topological $*$-probability spaces. Suppose there exists a bounded *-homomorphism $\Phi: A_{1} \rightarrow A_{2}$, and assume that

$$
\varphi_{2}(\Phi(a))=\varphi_{1}(a) \text {, for all } a \in A_{1} .
$$

Then, $\left(A_{1}, \varphi_{1}\right)$ is said to be free-homomorphic to $\left(A_{2}, \varphi_{2}\right)$. In particular, a $*$-homomorphism $\Phi$ satisfying (74) is called a free- $(*-)$ homomorphism from $\left(A_{1}, \varphi_{1}\right)$ to $\left(A_{2}, \varphi_{2}\right)$.

If $\Phi$ is a $*$-isomorphism satisfying (74), then it is called a free-isomorphism. If $\Phi$ is a free-isomorphism, then $\left(A_{1}, \varphi_{1}\right)$ is said to be free-isomorphic to $\left(A_{2}, \varphi_{2}\right)$.

By the free-homomorphic relation (74), one can get the following result.

Theorem 5. The n-prime shifts $G^{n}$ of (72) are free-homomorphisms on $\mathbb{L} \mathbb{S}$, for all $n \in \mathbb{N}_{0}$.

Proof. For any arbitrarily fixed $n \in \mathbb{N}_{0}$, take the $n$-prime shift $G^{n}$ on $\mathbb{L} \mathbb{S}$. Then, for any free reduced words $S$ of $\mathbb{L} S$ in the free semicircular family $\mathcal{X}$, one can get that

$$
\begin{aligned}
\tau^{0}\left(\left(G^{n}(S)\right)^{k}\right) & =\tau^{0}\left(S^{k}\right)=\tau^{0}\left(\left(S^{*}\right)^{k}\right) \\
& =\tau^{0}\left(\left(\left(G^{n}(S)\right)^{*}\right)^{k}\right),
\end{aligned}
$$

for all $k \in \mathbb{N}$, for all $n \in \mathbb{N}_{0}$, by (73) and the Möbius inversion. Indeed, the free distributions of free generators $\Theta_{p, j} \in \mathcal{X}$ of $\mathbb{L} \mathbb{S}$ are preserved by acting $G^{n}$, by (73).

Remark that, if $S=\prod_{l=1}^{N} \Theta_{p_{l}, j_{l}}^{n_{l}}$ is a free reduced word with its length- $N$, where either $\left(p_{1}, \ldots, p_{N}\right)$ is alternating in $\mathcal{P}$, or $\left(j_{1}, \ldots, j_{N}\right)$ is alternating in $\mathbb{Z}$, for $n_{1}, \ldots, n_{N} \in \mathbb{N}$, then the adjoint $S^{*}$ of $S$ is again a free reduced word with the same length- $N$,

$$
S^{*}=\prod_{l=1}^{N} \Theta_{p_{N-l+1}, j_{N-l+1}}^{n_{N-l+1}} \in \mathbb{L} \mathbb{S},
$$

by the self-adjointness of $\Theta_{p_{l}, j_{l}}$, for all $l=1, \ldots, N$. Therefore, the formula (75) holds by (73) under the Möbius inversion of [12], for all $n \in \mathbb{N}_{0}$.

By (61) and (63), all operators $T$ of $\mathbb{L} \mathbb{S}$ are the limits of linear combinations of free reduced words in $\mathcal{X}$. Thus, the free distributions of all summands of the operators $T$ of $\mathbb{L} \mathbb{S}$ are preserved by the $n$-prime shifts $G^{n}$ by (75), for all $n \in \mathbb{N}_{0}$. Therefore, the free distributions of $T$ are identical to the free distributions of $G^{n}(T)$, for all $n \in \mathbb{N}_{0}$, for all $T \in \mathbb{L} \mathbb{S}$. For example, the condition (74) is satisfied under the action of $G^{n}$ on $\mathbb{L} \mathbb{S}$. Equivalently, the $n$-prime shifts $G^{n}$ are free-homomorphisms on $\mathbb{L} \mathbb{S}$, for all $n \in \mathbb{N}_{0}$.

The above theorem says that our $n$-prime shifts $G^{n}$ are not only $*$-homomorphisms, but also free-homomorphisms on $\mathbb{L} \mathbb{S}$, for all $n \in \mathbb{N}_{0}$. 


\section{Shifts on $\mathbb{Z}$ Acting on $\mathbb{L S}$}

In this section, we consider certain shifting processes $h_{ \pm}$on $\mathbb{Z}$, and the corresponding *-homomorphisms $\beta_{ \pm}$on the semicircular Adelic filterization $\mathbb{L} \mathbb{S}$.

\subsection{Shifts $h_{ \pm}$on $\mathbb{Z}$}

Let $\mathbb{Z}$ be the set of all integers as usual. Define functions $h_{+}$and $h_{-}$on $\mathbb{Z}$ by the bijections on $\mathbb{Z}$,

$$
h_{+}(j)=j+1, \text { and } h_{-}(j)=j-1,
$$

for all $n \in \mathbb{Z}$. By the definition (76), one can have

$$
h_{+} \circ h_{-}=i d_{\mathbb{Z}}=h_{-} \circ h_{+}
$$

where $i d_{\mathbb{Z}}$ is the identity map on $\mathbb{Z}$, i.e.,

$$
h_{+}^{-1}=h_{-}, \text {and } h_{-}^{-1}=h_{+} \text {on } \mathbb{Z},
$$

by (77), where $f^{-1}$ is the inverse of $f$ (if exists).

Definition 14. Let $h_{ \pm}$be the bijections (76) on $\mathbb{Z}$. Then, we call $h_{ \pm}$, the $( \pm)$-shifts on $\mathbb{Z}$, respectively.

Let $h_{ \pm}$be the $( \pm)$-shifts (76) on $\mathbb{Z}$. Define the functions $h_{ \pm}^{n}$ on $\mathbb{Z}$ by

$$
h_{ \pm}^{n}=\underbrace{h_{ \pm} \circ h_{ \pm} \circ \cdots \circ h_{ \pm}}_{n \text {-times }}
$$

for all $n \in \mathbb{N}_{0}$, with axiomatization:

$$
h_{ \pm}^{0}=i d_{\mathbb{Z}} \text { on } \mathbb{Z}
$$

By the bijectivity (77) of $h_{ \pm}$, these functions $h_{ \pm}^{n}$ of (78) are bijective on $\mathbb{Z}$, too, for all $n \in \mathbb{N}_{0}$.

Definition 15. Let $h_{ \pm}^{n}$ be the bijections (78), for all $n \in \mathbb{N}_{0}$, where $h_{ \pm}$are the ( \pm )-shifts (77) on $\mathbb{Z}$. We call $h_{ \pm}^{n}$, the $n$-( \pm -shifts on $\mathbb{Z}$, for all $n \in \mathbb{N}_{0}$.

The $n$-( \pm )-shifts $h_{ \pm}^{n}$ of (78) are directly understood as bijections,

$$
h_{ \pm}^{n}(j)=j \pm n, \text { for all } j \in \mathbb{Z},
$$

for all $n \in \mathbb{N}_{0}$.

\subsection{Integer-Shift $*$-Homomorphisms on $\mathbb{L} \mathbb{S}$}

Let $h_{ \pm}^{n}$ be the $n$-( \pm -shifts (78) or (79) on $\mathbb{Z}$, for $n \in \mathbb{N}_{0}$. We now define $*$-homomorphisms $\beta_{ \pm}^{n}$ on the semicircular Adelic filterization $\mathbb{L} \mathbb{S}$ by the bounded "multiplicative" linear transformations satisfying

$$
\beta_{ \pm}^{n}\left(\Theta_{p, j}\right)=\Theta_{p, h_{ \pm}^{n}(j)}=\Theta_{p, j \pm n}
$$

for all $\Theta_{p, j} \in \mathcal{X}$, for all $n \in \mathbb{N}_{0}$, where $\mathcal{X}$ is the free semicircular family (60), generating $\mathbb{L} \mathbb{S}$.

It is easy to check that

$$
\begin{aligned}
\beta_{ \pm}^{n}\left(\left(t \Theta_{p, j}\right)^{*}\right) & =\bar{t} \beta_{ \pm}^{n}\left(\Theta_{p, j}\right)=\bar{t} \Theta_{p, j \pm n} \\
& =\left(t \Theta_{p, j \pm n}\right)^{*}=\left(\beta_{ \pm}^{n}\left(t \Theta_{p, j}\right)\right)^{*}
\end{aligned}
$$


for all $t \in \mathbb{C}, n \in \mathbb{N}_{0}$, and $\Theta_{p, j} \in \mathcal{X}$, in $\mathbb{L} \mathbb{S}$. Under linearity and multiplicativity of $\beta_{ \pm}^{n}$, it implies that

$$
\beta_{ \pm}^{n}\left(T^{*}\right)=\left(\beta_{ \pm}^{n}(T)\right)^{*}, \text { for all } T \in \mathbb{L} \mathbb{S} .
$$

For example, the morphisms $\beta_{ \pm}^{n}$ are $*$-homomorphisms on $\mathbb{L} \mathbb{S}$, for all $n \in \mathbb{N}_{0}$.

Definition 16. We call the $*$-homomorphisms $\beta_{ \pm}^{n}$ of (80), the $n$-( \pm -integer-shift(-*-homomorphism)s on $\mathbb{L} \mathbb{S}$, for all $n \in \mathbb{N}_{0}$. If $n=1$ in $\mathbb{N}_{0}$, we simply call $\beta_{ \pm}=\beta_{ \pm}^{1}$, the ( \pm -integer-shifts on $\mathbb{L} \mathbb{S}$.

For convenience, let

$$
\mathbb{N}_{0}^{ \pm} \stackrel{\text { def }}{=}\{ \pm\} \times \mathbb{N}_{0}
$$

from below.

Lemma 2. Let

$$
T=Q_{p, j}^{m} \text { and } S=\Theta_{p, j}^{m} \text { in } \mathbb{L} \mathbb{S},
$$

where $Q_{p, j} \in \mathcal{Q}, \Theta_{p, j} \in \mathcal{X}$, and $m \in \mathbb{N}$. Then,

$$
\begin{aligned}
\tau^{0}\left(\left(\beta_{e}^{n}\left(Q_{p, j}^{m}\right)\right)^{k}\right) & =\tau^{0}\left(\left(\left(\beta_{e}^{n}\left(Q_{p, j}^{m}\right)\right)^{*}\right)^{k}\right) \\
& =\omega_{k m} p_{1}^{k m\left(j_{1}+1\right)} c_{\frac{k m}{2}}=\tau^{0}\left(\left(Q_{p, j}^{m}\right)^{k}\right)
\end{aligned}
$$

and

$$
\begin{aligned}
\tau^{0}\left(\left(\beta_{e}^{n}\left(\Theta_{p, j}^{m}\right)\right)^{k}\right) & =\tau^{0}\left(\left(\left(\beta_{e}^{n}\left(\Theta_{p, j}^{m}\right)\right)^{*}\right)^{k}\right) \\
& =\omega_{k m} c_{\frac{k m}{2}}=\tau^{0}\left(\left(\Theta_{p, j}^{m}\right)^{k}\right)
\end{aligned}
$$

for all $k \in \mathbb{N}$, for all $(e, n) \in \mathbb{N}_{0}^{ \pm}$.

Proof. Let $T=Q_{q, k}^{m}$ and $S=\Theta_{q, k}^{m}$ be the free reduced words with their lengths- 1 in $\mathbb{L} \mathbb{S}$, for all $q \in \mathcal{P}, k \in$ $\mathbb{Z}$, for $m \in \mathbb{N}$. Then, by the $q^{2(k+1)}$-semicircularity of $Q_{q, k} \in \mathcal{Q}$, and by the semicircularity of $\Theta_{q, k} \in \mathcal{X}$, the operators $Q_{q, k}^{m}$ and $\Theta_{q, k}^{m}$ are self-adjoint in $\mathbb{L} \mathbb{S}$, for all $q \in \mathcal{P}, k \in \mathbb{Z}$, and $m \in \mathbb{N}$.

Thus, if $T=Q_{p, j}^{m}$ and $S=\Theta_{p, j}^{m}$ are in the sense of (81), then

$$
\beta_{e}^{n}(T)=Q_{p, j e n}^{m} \text { and } \beta_{e}^{n}(S)=\Theta_{p, j e n}^{m}
$$

are self-adjoint in $\mathbb{L} \mathbb{S}$ for all $(e, m) \in \mathbb{N}_{0}^{ \pm}$, where

$$
j e n= \begin{cases}j+n, & \text { if } e=+, \\ j-n, & \text { if } e=-\end{cases}
$$

Thus,

$$
\tau^{0}\left(\left(\beta_{e}^{n}(X)\right)^{k}\right)=\tau^{0}\left(\left(\left(\beta_{e}^{n}(X)\right)^{*}\right)^{k}\right)
$$

and

$$
\tau^{0}\left(\left(\beta_{e}^{n}(S)\right)^{k}\right)=\tau^{0}\left(\left(\left(\beta_{e}^{n}(S)\right)^{*}\right)^{k}\right)
$$


for all $k \in \mathbb{N}$.

Observe now that

$$
\begin{aligned}
\tau^{0}\left(\beta_{e}^{n}(X)^{k}\right) & =p^{k m(j+1)} \tau_{p, j e n}^{0}\left(\Theta_{p, j e n}^{k m}\right) \\
& =\omega_{k m} p_{1}^{k m\left(j_{1}+1\right)} c_{\frac{k n_{1}}{2}}=\tau^{0}\left(X^{k}\right)
\end{aligned}
$$

and

$$
\tau^{0}\left(\beta_{e}^{n}(S)^{k}\right)=\tau_{p, j e n}^{0}\left(\Theta_{p, j e n}^{k m}\right)=\omega_{k m} c_{\frac{k m}{2}}=\tau^{0}\left(S^{k}\right),
$$

by the semicircularity of $\Theta_{p, j e n} \in \mathcal{X}$ in $\mathbb{L S}$, for all $k \in \mathbb{N}$, and $(e, n) \in \mathbb{N}_{0}^{ \pm}$. Therefore, the formulas in (82) hold by (83) and (84).

The free-distributional data (82) demonstrate that the integer-shifts $\beta_{e}^{n}$ preserve the free distributions on $\mathbb{L} \mathbb{S}$ because they preserve the free distributions of generators of $\mathbb{L S}$ in $\mathcal{X}(\cup \mathcal{Q})$, for all $(e, n) \in \mathbb{N}_{0}^{ \pm}$.

Theorem 6. Let $\beta_{e}^{n}$ be an integer-shift on $\mathbb{L} \mathbb{S}$, for $(e, n) \in \mathbb{N}_{0}^{ \pm}$. Then, they are free-isomorphisms on the semicircular Adelic filterization $\mathbb{L} \mathbb{S}$.

Proof. Let $(e, n) \in \mathbb{N}_{0}^{ \pm}$, and let $\beta_{e}^{n}$ be an integer-shift on $\mathbb{L} \mathbb{S}$. Then, it is a well-defined $*$-isomorphism on $\mathbb{L S}$ because it is a generator-preserving, bijective $*$-homomorphism. Indeed, the restriction $\left.\beta_{e}^{n}\right|_{\mathcal{X}}$ is a bijection, as a function on the generator set $\mathcal{X}$ of $\mathbb{L} \mathbb{S}$. The bijectivity of $\beta_{e}^{n} \mid \mathcal{X}$ is guaranteed by the bijectivity of the $n$-( \pm -shifts $h_{ \pm}^{n}$ on $\mathbb{Z}$.

Moreover, by (82), the $*$-isomorphism $\beta_{e}^{n}$ preserves the free distributions of free generators of $\mathbb{L} \mathbb{S}$. Thus, the free distributions of all free reduced words of $\mathbb{L S}$ in $\mathcal{X}$ are preserved by $\beta_{e}^{n}$, by (82) and the Möbius inversion of [12]. Therefore, it preserves the free probability on $\mathbb{L} \mathbb{S}$ to that on $\beta_{e}^{n}(\mathbb{L} \mathbb{S})=\mathbb{L} \mathbb{S}$. Equivalently, the morphisms $\beta_{e}^{n}$ are free-isomorphisms on $\mathbb{L} \mathbb{S}$, by (74), for all $(e, n) \in \mathbb{N}_{0}^{ \pm}$.

\section{Shifts on $\mathcal{P} \times \mathbb{Z}$ and $*$-Homomorphisms on $\mathbb{L} \mathbb{S}$}

Before proceeding, let's remind that: if either $\left(p_{1}, \ldots, p_{N}\right)$, or $\left(j_{1}, \ldots, j_{N}\right)$ is alternating in $\mathcal{P}$, respectively, in $\mathbb{Z}$, for $N \in \mathbb{N}$, then

$$
X=\prod_{l=1}^{N} Q_{p_{l}, j_{l}}^{n_{l}} \text { and } Y=\prod_{l=1}^{N} \Theta_{p_{l}, j_{l}}^{n_{l}}
$$

are free reduced words with their lengths- $N$ in the semicircular Adelic filterization $\mathbb{L} \mathbb{S}$ (e.g., $[11,12,18])$, then

$$
\begin{aligned}
G^{n}(X) & =\left(\prod_{l=1}^{N} p_{l}^{n_{l}\left(j_{l}+1\right)}\right) G^{n}(Y) \\
& =\left(\prod_{l=1}^{N} p_{l}^{n_{l}\left(j_{l}+1\right)}\right)\left(\prod_{l=1}^{N} \Theta_{g^{(n)}\left(p_{l}\right), j_{l}}^{n_{l}}\right),
\end{aligned}
$$

and

by (71a), (71b) and (80), for all $n \in \mathbb{N}_{0}$, and $(e, n) \in \mathbb{N}_{0}^{ \pm}$, where $G^{n}$ are the $n$-prime shifts, and $\beta_{e}^{n}$ are the $n$-(e)-integer shifts on $\mathbb{L} \mathbb{S}$, which are free-homomorphisms. 


\subsection{Shifts on $\mathbb{P}=\mathcal{P} \times \mathbb{Z}$}

Now, define the Cartesian product set $\mathbb{P}$,

$$
\mathbb{P} \stackrel{\text { def }}{=} \mathcal{P} \times \mathbb{Z}
$$

Let $g^{n}$ be the $n$-shifts on $\mathcal{P}$, and let $h_{e}^{n}$ be the $n$-(e)-shifts on $\mathbb{Z}$, for $n \in \mathbb{N}_{0}$, and $(e, n) \in \mathbb{N}_{0}^{ \pm}$, with axiomatization:

$$
g^{0}=i d_{\mathcal{P}} \text {, and } h_{+}^{0}=i d_{\mathbb{Z}}=h_{-}^{0} .
$$

Define now shifts on the set $\mathbb{P}$ of (87) by

$$
s_{\left(e, n_{2}\right)}^{n_{1}} \stackrel{\text { def }}{=} g^{n_{1}} \times h_{e}^{n_{2}} \stackrel{\text { denote }}{=}\left(g^{n_{1}}, h_{e}^{n_{2}}\right),
$$

for all $n_{1}, n_{2} \in \mathbb{N}_{0}$, and $e \in\{ \pm\}$. For example, for any $(p, j) \in \mathbb{P}$,

$$
s_{\left(e, n_{2}\right)}^{n_{1}}(p, j)=\left(g^{n_{1}}(p), h_{e}^{n_{2}}(j)\right)=\left(g^{n_{1}}(p), j e n_{2}\right)
$$

in $\mathbb{P}$.

It is not difficult to check that such functions $s_{\left(e, n_{2}\right)}^{n_{1}}$ are injections on $\mathbb{P}$, since $g^{n_{1}}$ are injections on $\mathcal{P}$, and $h_{e}^{n_{2}}$ are bijections on $\mathbb{Z}$.

Definition 17. Let $s_{\left(e, n_{2}\right)}^{n_{1}}$ be injections (88) on the set $\mathbb{P}$ of $(87)$, for $n_{1} \in \mathbb{N}_{0}$, and $\left(e, n_{2}\right) \in \mathbb{N}_{0}^{ \pm}$, with identity,

$$
s_{(e, 0)}^{0}=i d_{\mathcal{P}} \times i d_{\mathbb{Z}}=i d_{\mathbb{P}}
$$

where $i d_{\mathbb{P}}$ means the identity map on $\mathbb{P}$. Then, these injections $s_{\left(e, n_{2}\right)}^{n_{1}}$ are called the shift(-function)s on $\mathbb{P}$.

\subsection{Prime-Integer Shifts on $\mathbb{L} \mathbb{S}$}

Let $\mathbb{P}$ be the Cartesian product set (87), and let $s_{\left(e, n_{2}\right)}^{n_{1}}$ be shifts (88) on $\mathbb{P}$. Then, for such shifts $s_{\left(e, n_{2}\right)}^{n_{1}}$, one can construct the corresponding $*$-homomorphisms $\sigma_{\left(e, n_{2}\right)}^{n_{1}}$ on the semicircular Adelic filterization $\mathbb{L} \mathbb{S}$, defined by

$$
\sigma_{\left(e, n_{2}\right)}^{n_{1}}=G^{n_{1}} \beta_{e}^{n_{2}} \text { on } \mathbb{L} \mathbb{S},
$$

for all $n_{1} \in \mathbb{N}_{0}$, and $\left(e, n_{2}\right) \in \mathbb{N}_{0}^{ \pm}$, where $G^{n_{1}}$ are the $n_{1}$-prime shifts, and $\beta_{e}^{n_{2}}$ are $n_{2}-(e)$-integer shifts on $\mathbb{L} \mathbb{S}$.

Since $G^{n_{1}}$ are free-homomorphisms, and $\beta_{e}^{n_{2}}$ are free-isomorphisms on $\mathbb{L} \mathbb{S}$, the morphism $\sigma_{\left(e, n_{2}\right)}^{n_{1}}$ of (89) are indeed well-defined $*$-homomorphisms on $\mathbb{L} \mathbb{S}$.

Proposition 8. Let $\sigma_{\left(e, n_{2}\right)}^{n_{1}}$ be a *-homomorphism (89) on LSS. Then,

$$
\sigma_{\left(e, n_{2}\right)}^{n_{1}}=\beta_{e}^{n_{2}} G^{n_{1}} \text { on } \mathbb{L} \mathbb{S},
$$

for all $n_{1} \in \mathbb{N}_{0},\left(e, n_{2}\right) \in \mathbb{N}_{0}^{ \pm}$.

Proof. By the very definition (89),

$$
\begin{gathered}
\sigma_{\left(e, n_{2}\right)}^{n_{1}}\left(\Theta_{p, j}\right)=G^{n_{1}}\left(\beta_{e}^{n_{2}}\left(\Theta_{p, j}\right)\right) \\
=G^{n_{1}}\left(\Theta_{p, j e n_{2}}\right)=\Theta_{g^{n_{1}}}(p), j e n_{2} \\
=\beta_{e}^{n_{2}}\left(\Theta_{g^{n_{1}}(p), j}\right)=\beta_{e}^{n_{2}}\left(G^{n_{1}}\left(\Theta_{p, j}\right)\right) \\
=\beta_{e}^{n_{2}} G^{n_{1}}\left(\Theta_{p, j}\right),
\end{gathered}
$$


for $\Theta_{p, j} \in \mathcal{X}$ of $\mathbb{L} \mathbb{S}$, for all $(p, j) \in \mathbb{P}$. Therefore, under multiplicativity of $\sigma_{\left(e, n_{2}\right)}^{n_{1}}$ on $\mathbb{L} \mathbb{S}$,

$$
\sigma_{\left(e, n_{2}\right)}^{n_{1}}(W) \stackrel{\text { def }}{=} G^{n_{1}} \beta_{e}^{n_{2}}(W)=\beta_{e}^{n_{2}} G^{n_{1}}(W),
$$

for all free reduced words $W$ of $\mathbb{L} \mathbb{S}$ in $\mathcal{X}$, by (86). It implies that the equality (90) holds on $\mathbb{L} \mathbb{S}$.

Let $\operatorname{Hom}(\mathbb{L} \mathbb{S})$ be the $(*-)$ homomorphism semigroup acting on the semicircular Adelic filterization $\mathbb{L} \mathbb{S}$, consisting of all $*$-homomorphisms on $\mathbb{L} \mathbb{S}$. Define a subset

$$
\sigma(\mathbb{L} S)=\left\{\sigma_{\left(e, n_{2}\right)}^{n_{1}}: n_{1}, n_{2} \in \mathbb{N}_{0}, e \in\{ \pm\}\right\}
$$

of $\operatorname{Hom}(\mathbb{L S})$, where $\sigma_{\left(e, n_{2}\right)}^{n_{1}}$ are in the sense of (89).

Definition 18. We call the $*$-homomorphisms $\sigma_{\left(e, n_{2}\right)}^{n_{1}}$ of (89), the prime-integer shift(-*-homomorphism)s on $\mathbb{L} \mathbb{S}$ (in short, pi-shifts on $\mathbb{L} \mathbb{S})$. For example, all elements of the set $\sigma(\mathbb{L S})$ of $(91)$ are called pi-shifts on $\mathbb{L} \mathbb{S}$.

Now, let's consider a structure theorem of the system $\sigma(\mathbb{L S})$ of $(91)$ in $\operatorname{Hom}(\mathbb{L} \mathbb{S})$.

Theorem 7. Let $\sigma(\mathbb{L} \mathbb{S})$ be the subset (91) of the homomorphism semigroup Hom $(\mathbb{L} \mathbb{S})$ acting on the semicircular Adelic filterization $\mathbb{L} \mathbb{S}$. Then,

(i) $\sigma(\mathbb{L S})$ is a commutative sub-monoid of $\operatorname{Hom}(\mathbb{L} \mathbb{S})$.

Proof. Let $\sigma(\mathbb{L S})$ be the subset $(91)$ contained in $\operatorname{Hom}(\mathbb{L S})$. Then, one can obtain that

$$
\sigma_{\left(e, n_{2}\right)}^{n_{1}} \sigma_{\left(r, k_{2}\right)}^{k_{1}}=\left(G^{n_{1}} \beta_{e}^{n_{2}}\right)\left(G^{k_{1}} \beta_{r}^{k_{2}}\right)
$$

by (89)

$$
=\left(G^{n_{1}} G^{k_{1}}\right)\left(\beta_{e}^{n_{2}} \beta_{r}^{k_{2}}\right)
$$

by $(90)$

$$
=G^{n_{1}+k_{1}} \beta_{s g n\left(e n_{2}+r k_{2}\right)}^{\left|e n_{2}+r k_{2}\right|}=\sigma_{\left(s g n\left(e n_{2}+r k_{2}\right),\left|e n_{2}+r k_{2}\right|\right)^{\prime}}^{n_{1}+k_{1}}
$$

where $\operatorname{sgn}$ is the sign map on $\mathbb{Z}$, satisfying

$$
\operatorname{sgn}(j)= \begin{cases}+ & \text { if } j \geq 0, \\ - & \text { if } j<0,\end{cases}
$$

for all $j \in \mathbb{Z}$, and $|$.$| is the absolute value on \mathbb{Z}$, for all $n_{1}, k_{1}, n_{2}, k_{2} \in \mathbb{N}_{0}$, and $e, r \in\{ \pm\}$.

The formula (92) shows that the product (or composition), inherited from that on $\operatorname{Hom}(\mathbb{L S})$, is closed on the set $\sigma(\mathbb{L S})$. Thus, one can consider $\sigma(\mathbb{L S})$ as an algebraic sub-structure $(\sigma(\mathbb{L} \mathbb{S})$, ·) in Hom $(\mathbb{L S})$.

Observe now that

$$
\begin{aligned}
& \left(\beta_{e_{1}}^{n_{1}} \beta_{e_{2}}^{n_{2}}\right) \beta_{e_{3}}^{n_{3}}=\beta_{s g n\left(e_{1} n_{1}+e_{2} n_{2}\right)}^{\left|e_{1} n_{1}+e_{2} n_{2}\right|} \beta_{e_{3}}^{n_{3}} \\
= & \beta_{s g n\left(e_{1} n_{1}+e_{2} n_{2}+e_{3} n_{3}\right)}^{\left|\left(e_{1} n_{1}+e_{2} n_{2}\right)+e_{3} n\right|}=\beta_{s g n\left(e_{1} n_{1}+e_{2} n_{2}+e_{3} n_{3}\right)}^{\left|e_{1} n_{1}+\left(e_{2} n_{2}+e_{3} n_{3}\right)\right|} \\
= & \beta_{e_{1}}^{n_{1}} \beta_{s g n\left(e_{2} n_{2}+e_{3} n_{3}\right)}^{\left|e_{2} n_{2}+e_{3} n_{3}\right|}=\beta_{e_{1}}^{n_{1}}\left(\beta_{e_{2}}^{n_{2}} \beta_{e_{3}}^{n_{3}}\right),
\end{aligned}
$$

on $\mathbb{L} \mathbb{S}$, for $\left(e_{l}, n_{l}\right) \in \mathbb{N}_{0}^{ \pm}$, for all $l=1,2$, 3. In addition, one has 


$$
\begin{aligned}
\left(G^{n_{1}} G^{n_{2}}\right) G^{n_{3}} & =G^{n_{1}+n_{2}} G^{n_{3}} \\
& =G^{n_{1}+n_{2}+n_{3}}=G^{n_{1}} G^{n_{2}+n_{3}} \\
& =G^{n_{1}}\left(G^{n_{2}} G^{n_{3}}\right)
\end{aligned}
$$

on $\mathbb{L} \mathbb{S}$, for all $n_{1}, n_{2}, n_{3} \in \mathbb{N}_{0}$.

Thus, one obtains that

by (92)

$$
\begin{aligned}
& \left(\sigma_{\left(e_{1}, k_{1}\right)}^{n_{1}} \sigma_{\left(e_{2}, k_{2}\right)}^{n_{2}}\right) \sigma_{\left(e_{3}, k_{3}\right)}^{n_{3}} \\
& =\sigma_{\left(s g n\left(e_{1} k_{1}+e_{2} k_{2}\right),\left|e_{1} k_{1}+e_{2} k_{2}\right|\right)}^{n_{1}+n_{2}} \sigma_{\left(e_{3}, k_{3}\right)}^{n_{3}} \\
& =\sigma_{\left(s g n\left(e_{1} k_{1}+e_{2} k_{2}+e_{3} k_{3}\right),|| e_{1} k_{1}+e_{2} k_{2}\left|+e_{3} k_{3}\right|\right)}^{\left(n_{1}+n_{2}\right)+n_{3}} \\
& =\sigma_{\left(\operatorname{sgn}\left(e_{1} k_{1}+e_{2} k_{2}+e_{3} k_{3}\right),\left|e_{1} k_{1}+\right| e_{2} k_{2}+e_{3} k_{3}||\right)}^{n_{1}+\left(n_{2}+n_{3}\right)} \\
& =\sigma_{\left(e_{1}, k_{1}\right)}^{n_{1}} \sigma_{\left(\operatorname{sgn}\left(e_{2} k_{2}+e_{3} k_{3}\right),\left|e_{2} k_{2}+e_{3} k_{3}\right|\right)}^{n_{2}+n_{3}} \\
& =\sigma_{\left(e_{1}, k_{1}\right)}^{n_{1}}\left(\sigma_{\left(e_{2}, k_{2}\right)}^{n_{2}} \sigma_{\left(e_{3}, k_{3}\right)}^{n_{3}}\right),
\end{aligned}
$$

by (90), (93) and (94), for $n_{l} \in \mathbb{N}_{0},\left(e_{l}, k_{l}\right) \in \mathbb{N}_{0}^{ \pm}$, for all $l=1,2$, 3. Thus, the operation (.) on $\sigma(\mathbb{L} \mathbb{S})$ is associative by $(95)$, and, hence, the algebraic pair $(\sigma(\mathbb{L S}), \cdot)$ forms a semigroup.

Definitely, one can take an element

$$
\sigma_{(e, 0)}^{0}=G^{0} \beta_{e}^{0}=1_{\mathbb{L S}} \cdot 1_{\mathbb{L S}}=1_{\mathbb{L S}} \in \sigma(\mathbb{L} \mathbb{S}),
$$

satisfying that

$$
\sigma_{(e, k)}^{n} \cdot 1_{\mathbb{L S}}=\sigma_{(e, k)}^{n}=1_{\mathbb{L S}} \cdot \sigma_{(e, k)}^{n} \text { in } \sigma(\mathbb{L S}),
$$

for all $n \in \mathbb{N}_{0}$, and $(e, k) \in \mathbb{N}_{0}^{ \pm}$.

Thus, the semigroup $(\sigma(\mathbb{L S}), \cdot)$ contains its $(\cdot)$-identity $1_{\mathbb{L S}}=\sigma_{(e, 0)}^{0}$ of $(96)$, and, hence, it is a well-defined monoid in $\operatorname{Hom}(\mathbb{L} \mathbb{S})$.

Finally, consider that

$$
\begin{aligned}
& G^{n_{1}} G^{n_{2}}=G^{n_{1}+n_{2}}=G^{n_{2}+n_{1}}=G^{n_{2}} G^{n_{1}}, \\
& \text { and } \\
& \beta_{e_{1}}^{k_{1}} \beta_{e_{2}}^{k_{2}}=\beta_{\text {sgn }\left(e_{1} k_{1}+e_{2} k_{2}\right)}^{\left|e_{1} k_{1}+e_{2} k_{2}\right|}=\beta_{\text {sgn }\left(e_{2} k_{2}+e_{1} k_{1}\right)}^{\left|e_{2} k_{2}+e_{1} k_{1}\right|}=\beta_{e_{2}}^{k_{2}} \beta_{e_{1}}^{k_{1}},
\end{aligned}
$$

on $\mathbb{L} \mathbb{S}$, for all $n_{1}, n_{2} \in \mathbb{N}_{0}$, and $\left(e_{1}, k_{1}\right),\left(e_{2}, k_{2}\right) \in \mathbb{N}_{0}^{ \pm}$.

Therefore,

$$
\begin{aligned}
\sigma_{\left(e_{1}, k_{1}\right)}^{n_{1}} \sigma_{\left(e_{2}, k_{2}\right)}^{n_{2}} & =\sigma_{\left(\operatorname{sgn}\left(e_{1} k_{1}+e_{2} k_{2}\right),\left|e_{1} k_{1}+e_{2} k_{2}\right|\right)}^{n_{1}+n_{2}} \\
& =\sigma_{\left(e_{2}, k_{2}\right)}^{n_{2} \sigma_{\left(e_{1}, k_{1}\right)^{\prime}}^{n_{1}}}
\end{aligned}
$$

on $\mathbb{L} \mathbb{S}$, for all $n_{1}, n_{2} \in \mathbb{N}_{0}$, and $\left(e_{1}, k_{1}\right),\left(e_{2}, k_{2}\right) \in \mathbb{N}_{0}^{ \pm}$, by (90) and (97).

Thus, the monoid $(\sigma(\mathbb{L S}), \cdot)$ is commutative by (98). Therefore, the system $\sigma(\mathbb{L S})$ of $(91)$ is a commutative sub-monoid of the homomorphism semigroup Hom $(\mathbb{L} \mathbb{S})$.

Remark that, even though free-isomorphisms $\beta_{e}^{n_{2}}$ have their inverses

$$
\left(\beta_{e}^{n_{2}}\right)^{-1}=\beta_{-e}^{n_{2}} \text { in } \operatorname{Hom}(\mathbb{L} \mathbb{S}), \forall\left(e, n_{2}\right) \in \mathbb{N}_{0}^{ \pm},
$$


the free-homomorphisms $G^{n_{1}}$ are not invertible in $\operatorname{Hom}(\mathbb{L} \mathbb{S})$, in general, for $n_{1} \in \mathbb{N}_{0}$. In particular, $G^{n_{1}}$ is bijective, if and only if $n_{1}=0$ in $\mathbb{N}_{0}$. Therefore, the $*$-homomorphisms $\sigma_{\left(e, n_{2}\right)}^{n_{1}}$ are not invertible in $\operatorname{Hom}(\mathbb{L} \mathbb{S})$, whenever $n_{1} \in \mathbb{N}$, for all $\left(e, n_{2}\right) \in \mathbb{N}_{0}^{ \pm}$. Thus, the commutative monoid $\sigma(\mathbb{L} \mathbb{S})$ cannot be a group in $\operatorname{Hom}(\mathbb{L} \mathbb{S})$.

The above structure theorem characterizes the algebraic structure of $\sigma(\mathbb{L} \mathbb{S})$ as a commutative monoid embedded in $\operatorname{Hom}(\mathbb{L} \mathbb{S})$.

Definition 19. Let $\sigma(\mathbb{L S})$ be a commutative sub-monoid (91) of the homomorphism semigroup Hom $(\mathbb{L} \mathbb{S})$. Then, this monoid $\sigma(\mathbb{L S})$ is called the prime-integer-shift monoid (in short, the pi-shift monoid) on $\mathbb{L} \mathbb{S}$.

\subsection{Free-Distributional Data on $\mathbb{L} \mathbb{S}$ Affected by $\sigma(\mathbb{L} \mathbb{S})$}

Recall that the prime-shifts $G^{n}$ are injective free-homomorphisms on $\mathbb{L} \mathbb{S}$ by (75), for all $n \in \mathbb{N}_{0}$; and the integer-shifts $\beta_{e}^{n}$ are free-isomorphisms on $\mathbb{L} \mathbb{S}$ by $(82)$, for all $(e, n) \in \mathbb{N}_{0}^{ \pm}$. Thus, it is not difficult to verify that the pi-shifts $\sigma_{(e, k)}^{n} \in \sigma(\mathbb{L} \mathbb{S})$ preserves the free probability on $\mathbb{L} \mathbb{S}$, for all $n \in \mathbb{N}_{0}$ and $(e, k) \in \mathbb{N}_{0}^{ \pm}$.

Theorem 8. Let $Q_{p, j} \in \mathcal{Q}$, and $\Theta_{p, j} \in \mathcal{X}$ in $\mathbb{L} \mathbb{S}$, for $p \in \mathcal{P}, j \in \mathbb{Z}$, and let $\sigma \stackrel{\text { denote }}{=} \sigma_{(e, k)}^{n}$ be a pi-shift in the pi-shift monoid $\sigma(\mathbb{L} \mathbb{S})$, for $n \in \mathbb{N}_{0},(e, k) \in \mathbb{N}_{0}^{ \pm}$. Then, $\sigma\left(Q_{p, j}\right)$ is $p^{2(j+1)}$-semicircular in $\mathbb{L} \mathbb{S}$, and $\sigma\left(\Theta_{p, j}\right)$ is semicircular in $\mathbb{L} \mathbb{S}$. For example,

$$
\text { All pi-shifts in } \sigma(\mathbb{L S}) \text { are injective free-homomorphism on } \mathbb{L} \mathbb{S} \text {. }
$$

Proof. Let $\sigma \in \sigma(\mathbb{L} \mathbb{S})$ be a pi-shift given as above. Then,

$$
\begin{aligned}
\tau^{0}\left(\sigma\left(Q_{p, j}\right)^{s}\right) & =\tau^{0}\left(\sigma\left(Q_{p, j}^{s}\right)\right) \\
& =p^{s(j+1)} \tau^{0}\left(\Theta_{g^{n}}^{s}(p), j e k\right) \\
& =p^{s(j+1)}\left(\omega_{s} \mathcal{c}_{\frac{s}{2}}\right)=\tau^{0}\left(Q_{p, j}^{s}\right)
\end{aligned}
$$

and

for all $s \in \mathbb{N}$.

Therefore, $\sigma\left(Q_{p, j}\right)$ (resp., $\left.\sigma\left(\Theta_{p, j}\right)\right)$ is $p^{2(j+1)}$-semicircular (resp., semicircular) in $\mathbb{L} \mathbb{S}$, too.

By (100) and the Möbius inversion of [12], the free distributions of all free reduced words of $\mathbb{L} \mathbb{S}$ in the free semicircular family $\mathcal{X}$ are preserved by pi-shifts of $\sigma(\mathbb{L} \mathbb{S})$. It shows that all pi-shifts preserves the free probability on $\mathbb{L} \mathbb{S}$. Therefore, the statement (99) holds.

\section{Prime-Integer-Shift Operators on $\mathbb{L} \mathbb{S}$}

Let $\mathbb{L} \mathbb{S}$ be the semicircular Adelic filterization, and $\sigma(\mathbb{L S})$, the pi-shift monoid (91) consisting of all pi-shifts $\sigma_{(e, k)}^{n}$, which are injective free-homomorphisms on $\mathbb{L} \mathbb{S}$ by (99), for all $n \in \mathbb{N}_{0},(e, k) \in \mathbb{N}_{0}^{ \pm}$. In this section, we understand the Banach $*$-algebra $\mathbb{L} \mathbb{S}$ as a Banach space, and construct the operator space $B(\mathbb{L} \mathbb{S})$ consisting of all bounded linear transformations on this Banach space $\mathbb{L} \mathbb{S}$ (e.g., [2]).

Since all monoidal elements $\sigma_{(e, n)}^{n} \in \sigma(\mathbb{L} \mathbb{S})$ are injective $*$-homomorphisms, they are bounded linear transformations on $\mathbb{L} \mathbb{S}$, i.e., they can be regarded as Banach-space operators in $B(\mathbb{L} \mathbb{S})$. For example,

$$
\sigma(\mathbb{L} \mathbb{S}) \subset \operatorname{Hom}(\mathbb{L} \mathbb{S}) \subset B(\mathbb{L} \mathbb{S})
$$


Now, let's consider the following bounded "multiplicative" linear transformation $G^{*}$ on $\mathbb{L} \mathbb{S}$, defined by

$$
G^{*}\left(\Theta_{p, j}\right) \stackrel{\text { def }}{=} \begin{cases}\Theta_{q, j} & \text { if } \exists q \in \mathcal{P} \text { s.t., } g(q)=p, \\ 0_{\mathbb{L} \mathbb{S}} & \text { otherwise, }\end{cases}
$$

for all semicircular elements $\Theta_{p, j} \in \mathcal{X}$. For instance,

$$
G^{*}\left(\Theta_{3, j}\right)=\Theta_{2, j}, G^{*}\left(\Theta_{17, j}\right)=\Theta_{13, j},
$$

and

$$
G^{*}\left(\Theta_{2, j}\right)=0_{\mathbb{L} \mathbb{S}}
$$

etc., for all $j \in \mathbb{Z}$. Observe that, for any $t \in \mathbb{C}$,

$$
\begin{aligned}
G^{*}\left(\left(t \Theta_{p, j}\right)^{*}\right) & =G^{*}\left(\bar{t} \Theta_{p, j}\right) \\
& = \begin{cases}\bar{t} \Theta_{q, j}=\left(G^{*}\left(t \Theta_{p, j}\right)\right)^{*} & \text { if } g(q)=p \text { in } \mathcal{P}, \\
\bar{t} 0_{\mathbb{L} \mathbb{S}}=\left(G^{*}\left(t \Theta_{p, j}\right)\right)^{*} & \text { otherwise, }\end{cases}
\end{aligned}
$$

for all $\Theta_{p, j} \in \mathcal{X}$, implying that

$$
G^{*}\left(T^{*}\right)=\left(G^{*}(T)\right)^{*}, \text { for all } T \in \mathbb{L} \mathbb{S},
$$

i.e., this multiplicative linear transformation $G^{*}$ of $(102)$ is a $*$-homomorphism on $\mathbb{L} \mathbb{S}$, i.e.,

$$
G^{*} \in \operatorname{Hom}(\mathbb{L} \mathbb{S}) .
$$

Thus, one can define the $n$-iterated products $\left(G^{*}\right)^{n}$ of $G^{*}$ in $\operatorname{Hom}(\mathbb{L S})$,

$$
\left(G^{*}\right)^{n}=\underbrace{G^{*} G^{*} \cdots G^{*}}_{n \text {-times }} \in \operatorname{Hom}(\mathbb{L S}),
$$

for all $n \in \mathbb{N}$.

For example, the $*$-homomorphism $\left(G^{*}\right)^{3}$ in the sense of (103) satisfies that

$$
\left(G^{*}\right)^{3}\left(\Theta_{2, j}\right)=\left(G^{*}\right)^{3}\left(\Theta_{3, j}\right)=\left(G^{*}\right)^{3}\left(\Theta_{5, j}\right)=0_{\mathbb{L} \mathbb{S}},
$$

and

$$
\left(G^{*}\right)^{3}\left(\Theta_{7, j}\right)=\Theta_{2, j}
$$

more generally,

$$
\left(G^{*}\right)^{3}\left(\Theta_{p, j}\right)=\Theta_{q, j}, \text { if } g^{3}(q)=p \text { in } \mathcal{P},
$$

for all $p \in \mathcal{P}$. Thus, it is non-zero if and only if $p>5$. Here, the quantity 5 is the " 3 -rd" prime in the TOset $\mathcal{P}$ of (67). 
Proposition 9. Let $\left(G^{*}\right)^{n}$ be a $*$-homomorphism (103), and let $G^{n}$ be the n-prime shift (72) in Hom $(\mathbb{L} \mathbb{S})$, for $n \in$ $\mathbb{N}_{0}$. Then,

$$
\begin{aligned}
& \left(G^{*}\right)^{n} G^{n}=1_{\mathbb{L S}, \text { on } \mathbb{L S},} \\
& \text { and } \\
& G^{n}\left(G^{*}\right)^{n}\left(\Theta_{p, j}\right)= \begin{cases}\Theta_{p, j}, & \text { if } \exists q \in \mathcal{P}, \text { s.t. }, \\
g^{n}(q)=p \text { in } \mathcal{P}, \\
0_{\mathbb{L S},}, & \text { otherwise, }\end{cases}
\end{aligned}
$$

for all semicircular elements $\Theta_{p, j} \in \mathcal{X}$, for all $n \in \mathbb{N}$.

Proof. The formula (104) is proven by the definition (102) and (103).

Now, let

$$
\mathcal{G}_{0}^{*}=\left\{\left(G^{*}\right)^{n} \in \operatorname{Hom}(\mathbb{L} \mathbb{S}): n \in \mathbb{N}_{0}\right\} \cup\left\{0_{\mathbb{L S}}\right\}
$$

with axiomatization:

$$
\left(G^{*}\right)^{0}=1_{\mathbb{L S}} \in \operatorname{Hom}(\mathbb{L S}),
$$

where $\left(G^{*}\right)^{n}$ are in the sense of (103), for all $n \in \mathbb{N}$.

Now, define the subset $\mathcal{G}$ of $\operatorname{Hom}(\mathbb{L} \mathbb{S})$, consisting of all our $n$-prime shifts $G^{n}$ of (72), for all $n \in \mathbb{N}_{0}$, i.e.,

$$
\mathcal{G}=\left\{G^{n}: n \in \mathbb{N}_{0}\right\} .
$$

Note that both the sets $\mathcal{G}_{0}^{*}$ of (105), and $\mathcal{G}$ of (106) form semigroups in $\operatorname{Hom}(\mathbb{L} \mathbb{S})$. Indeed,

$$
\begin{aligned}
\left(\left(G^{*}\right)^{n_{1}}\left(G^{*}\right)^{n_{2}}\right)\left(G^{*}\right)^{n_{3}} & =\left(G^{*}\right)^{n_{1}+n_{2}+n_{3}} \\
& =\left(G^{*}\right)^{n_{1}}\left(\left(G^{*}\right)^{n_{2}}\left(G^{*}\right)^{n_{3}}\right),
\end{aligned}
$$

in $\mathcal{G}_{0}^{*}$ by (103) and (104), and

$$
\left(G^{n_{1}} G^{n_{2}}\right) G^{n_{3}}=G^{n_{1}+n_{2}+n_{3}}=G^{n_{1}}\left(G^{n_{2}} G^{n_{3}}\right),
$$

by (106).

Thus, one can define the direct product semigroup $\mathfrak{G}_{0}$ of $\mathcal{G}_{0}^{*}$ and $\mathcal{G}$,

$$
\mathfrak{G}_{0} \stackrel{\text { def }}{=} \mathcal{G}_{0}^{*}+\mathcal{G} \text { in } \operatorname{Hom}(\mathbb{L S}),
$$

under the inherited operation $(\cdot)$ on $\operatorname{Hom}(\mathbb{L S})$.

For example, this algebraic sub-structure $\mathfrak{G}_{0}$ of (107) satisfies

$$
\mathfrak{G}_{0}=\mathcal{G}_{0}^{*} \sqcup \mathcal{G} \text {, set-theoretically, }
$$

satisfying

$$
\left(F_{1}, F_{2}\right) \in \mathfrak{G}_{0} \times \mathfrak{G}_{0} \longmapsto F_{1} F_{2} \in \mathfrak{G}_{0},
$$


as a sub-semigroup of $\operatorname{Hom}(\mathbb{L} \mathbb{S})$.

Indeed, the operation $(\cdot)$ on $\mathfrak{G}_{0}$ satisfies that

$$
\left(G^{*}\right)^{n_{1}} G^{n_{2}}\left(\Theta_{p, j}\right)= \begin{cases}\Theta_{p, j} & \text { if } n_{1}=n_{2} \\ \left(G^{*}\right)^{n_{1}-n_{2}}\left(\Theta_{p, j}\right) & \text { if } n_{1}>n_{2} \\ G^{n_{2}-n_{1}}\left(\Theta_{p, j}\right) & \text { if } n_{1}<n_{2}\end{cases}
$$

and

$$
G^{n_{1}}\left(G^{*}\right)^{n_{2}}\left(\Theta_{p, j}\right)= \begin{cases} & \text { if } n_{1}=n_{2} \text {, and } \\ \Theta_{p, j} & \text { if } \exists q \in \mathcal{P} \text {, such that } \\ & g^{n_{2}}(q)=p \text { in } \mathcal{P} \\ & \text { if } n_{1}>n_{2} \text {, and } \\ G^{n_{1}-n_{2}}\left(\Theta_{p, j}\right) & \text { if } \exists q \in \mathcal{P}, \text { such that } \\ & g^{n_{2}}(q)=p \text { in } \mathcal{P} \\ & \text { if } n_{1}<n_{2} \text {, and } \\ & \text { if } \exists q \in \mathcal{P} \text {, such that } \\ g^{n_{2}}(q)=p \text { in } \mathcal{P} \\ G^{n_{1}}\left(\Theta_{q, j}\right) \\ & \text { otherwise, }\end{cases}
$$

by (103) and (104).

Since this sub-semigroup $\mathfrak{G}_{0}$ of (107) contains $0_{\mathbb{L} \mathbb{S}}$, having no (.)-inverse in $\mathfrak{G}_{0}$, it is a sub-semigroup of $\operatorname{Hom}(\mathbb{L S})$, which is not a group. Moreover, this semigroup $\mathfrak{G}_{0}$ is "not" commutative by (108).

Definition 20. The direct-product, noncommutative sub-semigroup $\mathfrak{G}_{0}=\mathcal{G}_{0}^{*}+\mathcal{G}$ of (107) is called the prime-shift semigroup of $\operatorname{Hom}(\mathbb{L S})$.

Define now the subset $\sum(\mathbb{L} \mathbb{S})$ of $\operatorname{Hom}(\mathbb{L} \mathbb{S})$ by

$$
\Sigma(\mathbb{L S}) \stackrel{\text { def }}{=}\left\{\begin{array}{l|l}
K \beta_{e}^{n} \in \operatorname{Hom}(\mathbb{L} \mathbb{S}) \mid \begin{array}{c}
K \in \mathfrak{G}_{0}, \\
\forall(e, n) \in \mathbb{N}_{0}^{ \pm}
\end{array}
\end{array}\right\},
$$

where $\beta_{e}^{n}$ are the $n$-(e)-integer shifts on $\mathbb{L} \mathbb{S}$, for all $(e, n) \in \mathbb{N}_{0}^{ \pm}$.

Note that our pi-shift monoid $\sigma(\mathbb{L S})$ is contained in the set $\sum(\mathbb{L S})$ of $(109)$. Note also that the elements

$$
\sigma_{(e, k)}^{n}=G^{n} \beta_{e}^{k} \in \sigma(\mathbb{L} \mathbb{S}) \subset \sum(\mathbb{L} \mathbb{S})
$$

are free-homomorphisms, but the elements

$$
\left(G^{*}\right)^{n} \beta_{e}^{k} \in\left(\sum(\mathbb{L} \mathbb{S}) \backslash \sigma(\mathbb{L} \mathbb{S})\right) \subset \sum(\mathbb{L} \mathbb{S})
$$

cannot be free-homomorphisms by (102), (106) and (108), in general. Especially, if $n \neq 0$ in $\mathbb{N}_{0}$, then they are $*$-homomorphisms, which are not free-homomorphisms.

Observe now that if $G^{*} \in \mathcal{G}_{0}^{*} \subset \mathfrak{G}_{0}$, and, hence, if

$$
G^{*} \beta_{e}^{n}, \beta_{e}^{n} G^{*} \in \operatorname{Hom}(\mathbb{L} \mathbb{S}),
$$

then 


$$
G^{*} \beta_{e}^{n}\left(\Theta_{p, j}\right)=G^{*}\left(\Theta_{p, j e n}\right)=\left\{\begin{aligned}
\Theta_{q, j e n} & \text { if } \exists q \in \mathcal{P}, \text { s.t., } \\
& g(q)=p \text { in } \mathcal{P} \\
0_{\mathbb{L S}} & \text { otherwise, }
\end{aligned}\right.
$$

and

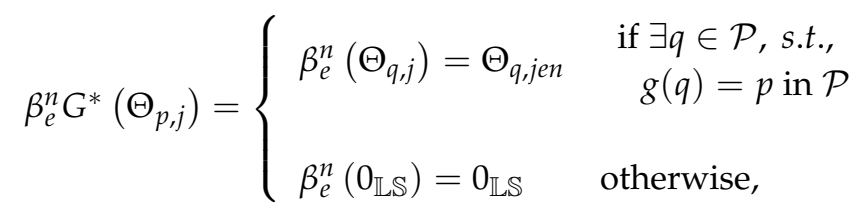

for all $\Theta_{p, j} \in \mathcal{X}$, implying that

$$
G^{*} \beta_{e}^{n}=\beta_{e}^{n} G^{*} \text { in } \operatorname{Hom}(\mathbb{L} \mathbb{S})
$$

by (63). Therefore,

$$
G^{* n_{1}} \beta_{e}^{n_{2}}=\beta_{e}^{n_{2}} G^{* n_{1}} \text { in } \operatorname{Hom}(\mathbb{L} \mathbb{S}),
$$

for all $n_{1}, n_{2} \in \mathbb{N}, e \in\{ \pm\}$, by the induction on (110).

Theorem 9. The subset $\sum(\mathbb{L} \mathbb{S})$ of (109) is a noncommutative sub-semigroup of the homomorphism semigroup $\operatorname{Hom}(\mathbb{L} \mathbb{S})$.

Proof. Let $\sum(\mathbb{L S})$ be the subset (109) of $\operatorname{Hom}(\mathbb{L S})$. Then, for any $K_{1} \beta_{e_{1}}^{n_{1}}, K_{2} \beta_{e_{2}}^{n_{2}} \in \sum(\mathbb{L} \mathbb{S})\left(\right.$ for $\left.K_{1}, K_{2} \in \mathfrak{G}_{0}\right)$, one has that

$$
\begin{aligned}
& \left(K_{1} \beta_{e_{1}}^{n_{1}}\right)\left(K_{2} \beta_{e_{2}}^{n_{2}}\right)=K_{1}\left(\beta_{e_{1}}^{n_{1}} K_{2}\right) \beta_{e_{2}}^{n_{2}} \\
= & K_{1} K_{2} \beta_{e_{1}}^{n_{1}} \beta_{e_{2}}^{n_{2}}=\left(K_{1} K_{2}\right)\left(\beta_{\text {sgn }\left(e_{1} n_{1}+e_{2} n_{2}\right)}^{\left|e_{1} n_{1}+e_{2} n_{2}\right|}\right),
\end{aligned}
$$

with $K_{1} K_{2} \in \mathfrak{G}_{0}$, by (90) and (111). Thus, the inherited product (.) on $\operatorname{Hom}(\mathbb{L} \mathbb{S})$ is closed in $\sum(\mathbb{L} \mathbb{S})$.

In addition, by (90), (106), (108) and (111), we have

$$
\left(\left(K_{1} \beta_{e_{1}}^{n_{1}}\right)\left(K_{2} \beta_{e_{2}}^{n_{2}}\right)\right)\left(K_{3} \beta_{e_{3}}^{n_{3}}\right)=\left(K_{1} \beta_{e_{1}}^{n_{1}}\right)\left(\left(K_{2} \beta_{e_{2}}^{n_{2}}\right)\left(K_{3} \beta_{e_{3}}^{n_{3}}\right)\right),
$$

in $\sum(\mathbb{L} \mathbb{S})$. Therefore, the algebraic pair $\left(\sum(\mathbb{L} \mathbb{S}), \cdot\right)$ forms a sub-semigroup of $\operatorname{Hom}(\mathbb{L} \mathbb{S})$.

It is trivial that $\sum(\mathbb{L S})$ is not commutative by (106) and (108).

Definition 21. Let $\sum(\mathbb{L S})$ be a noncommutative sub-semigroup (109) of Hom $(\mathbb{L S})$. We call $\sum(\mathbb{L S})$, the prime-integer-shift-operator semigroup (in short, the pi-shift-operator semigroup).

Note again that the pi-shift-operator semigroup $\sum(\mathbb{L} \mathbb{S})$ is contained in $\operatorname{Hom}(\mathbb{L} \mathbb{S})$, and, hence, it is contained in the operator space $B(\mathbb{L} \mathbb{S})$ by $(101)$, as operators acting on the Banach space $\mathbb{L} \mathbb{S}$. Therefore, one can construct the subspace $\mathcal{S}\left(\sum(\mathbb{L} \mathbb{S})\right)$ of the operator space $B(\mathbb{L S})$ spanned by $\sum(\mathbb{L} \mathbb{S})$,

$$
\mathcal{S} \stackrel{\text { denote }}{=} \mathcal{S}\left(\sum(\mathbb{L} \mathbb{S})\right) \stackrel{\text { def }}{=} \operatorname{span}_{\mathbb{C}}\left(\sum(\mathbb{L} \mathbb{S})\right)
$$

in $B(\mathbb{L S})$, where $\operatorname{span}_{\mathbb{C}}(Z)$ are the subspaces of $B(\mathbb{L S})$ generated by subsets $Z$ of $B(\mathbb{L S})$.

For the subspace $\mathcal{S}$ of (112), define an (pure-algebraic) algebra $\mathcal{A}_{a}$ by

$$
\mathcal{A}_{a}=\mathbb{C}[\mathcal{S}] \text { in } B(\mathbb{L} \mathbb{S})
$$


By the above theorem, such an algebra $\mathcal{A}_{a}$ of (113) is well-defined in $B(\mathbb{L} \mathbb{S})$. Thus, one can define the topological closure $\mathcal{A}\left(\sum(\mathbb{L S})\right)$ of $\mathcal{A}_{a}$ by

$$
\mathcal{A}\left(\sum(\mathbb{L} \mathbb{S})\right) \stackrel{\text { denote }}{=} \mathcal{A} \stackrel{\text { def }}{=} \overline{\mathcal{A}_{a}} \text { in } B(\mathbb{L} \mathbb{S}),
$$

where $\overline{\mathcal{Y}}$ are the topological closures of subsets $\mathcal{Y}$ of $B(\mathbb{L S})$, where $\mathcal{A}_{a}$ is an algebra (113).

Then, this sub-structure $\mathcal{A}$ of (114) is not only a closed subspace of $B(\mathbb{L} \mathbb{S})$, but also a well-defined Banach algebra embedded in the operator space $B(\mathbb{L} \mathbb{S})$.

Definition 22. Let $\mathcal{A} \stackrel{\text { denote }}{=} \mathcal{A}\left(\sum(\mathbb{L} S)\right)$ be the Banach algebra (114) in $B(\mathbb{L S})$, generated by the pi-shift-operator semigroup $\sum(\mathbb{L} \mathbb{S})$. Then, we call $\mathcal{A}$, the pi-shift-operator (Banach-)algebra. In addition, all elements of $\mathcal{A}$ are said to be pi-shift operators (acting) on $\mathbb{L} \mathbb{S}$.

By the definition (112), every pi-shift operators $T \in \mathcal{S} \subset \mathcal{A}$ have its expression,

$$
T=\sum_{r \in\{1, *\}, n \in \mathbb{N}_{0},(e, k) \in \mathbb{N}_{0}^{ \pm}} t_{(e, k)}^{r n} \sigma_{(e, k)^{\prime}}^{r n}
$$

with coefficients,

$$
t_{(e, k)}^{r n} \in \mathbb{C}
$$

where $\sum$ is a finite sum (under topology).

By (113) and (114), all pi-shift operators of $\mathcal{A}$ are the limits of linear combinations of "products" of such operators $T$ in the sense of (115). For example, the operators formed by $T$ of (115) generate the pi-shift operator algebra $\mathcal{A}$.

For convenience, define a Cartesian product set $\mathbb{N}_{0}^{* \pm}$ by

$$
\begin{aligned}
\mathbb{N}_{0}^{* \pm} & \stackrel{\text { def }}{=}\{1, *\} \times \mathbb{N}_{0} \times \mathbb{N}_{0}^{ \pm} \\
& =\{1, *\} \times \mathbb{N}_{0} \times\left(\{ \pm\} \times \mathbb{N}_{0}\right) .
\end{aligned}
$$

Then, by using this new notation (116), the expression (115) can be re-expressed by

$$
T=\sum_{(r, n,(e, k)) \in \mathbb{N}_{0}^{* \pm}} t_{(e, k)}^{r n} \sigma_{(e, k)}^{r n} \in \mathcal{S}
$$

in $\mathcal{A}$. Now, on the pi-shift-operator algebra $\mathcal{A}$, define a unary operation $(*)$ by the operation

$$
T \in \mathcal{A} \longmapsto T^{*} \in \mathcal{A},
$$

satisfying

$$
\left(t \sigma_{(e, k)}^{r n}\right)^{*}=\bar{t} \sigma_{(-e, k)}^{(* r) n} i n \mathcal{A}
$$

for all $t \in \mathbb{C}$ and $\sigma_{(e, k)}^{r n} \in \sum(\mathbb{L S})$, where

$$
* r= \begin{cases}* & \text { if } r=1 \\ * *=1 & \text { if } r=*\end{cases}
$$

for all $r \in\{1, *\}$, whenever $T$ is in the sense of (115) in $\mathcal{S} \subset \mathcal{A}$.

Then, this operation $(*)$ of $(117)$ satisfies the followings. First,

$$
\left(\left(\sigma_{(e, k)}^{r n}\right)^{*}\right)^{*}=\left(\sigma_{(-e, k)}^{* r n}\right)^{*}=\sigma_{(-(-e), k)}^{* * r n}=\sigma_{(e, k)}^{r n},
$$


in $\mathcal{A}$, for all $(r, n,(e, k)) \in \mathbb{N}_{0}^{* \pm}$. Thus, by (114) and (117),

$$
T^{* *}=T \text { in } \mathcal{A} \text {, for all } T \in \mathcal{A} .
$$

If $\left(r_{l}, n_{l},\left(e_{l}, k_{l}\right)\right) \in \mathbb{N}_{0}^{* \pm}$, for $l=1,2$, then

$$
\begin{aligned}
\left(\sigma_{\left(e_{1}, k_{1}\right)}^{r_{1} n_{1}}+\sigma_{\left(e_{2}, k_{2}\right)}^{r_{2} n_{2}}\right)^{*} & =\sigma_{\left(-e_{1}, k_{1}\right)}^{\left(* r_{1}\right) n_{1}}+\sigma_{\left(-e_{2}, k_{2}\right)}^{\left(* r_{2}\right) n_{2}} \\
& =\left(\sigma_{\left(e_{1}, k_{1}\right)}^{r_{1} n_{1}}\right)^{*}+\left(\sigma_{\left(e_{2}, k_{2}\right)}^{r_{2} n_{2}}\right)^{*},
\end{aligned}
$$

in $\mathcal{A}$, by (117). Thus, if $T_{1}, T_{2} \in \mathcal{A}$ are in the sense of (115), then

$$
\left(T_{1}+T_{2}\right)^{*}=T_{1}^{*}+T_{2}^{*} \text { in } \mathcal{A},
$$

by (114).

Now, observe that

$$
\begin{gathered}
\left(\sigma_{\left(e_{1}, k_{1}\right)}^{r_{1} n_{1}} \sigma_{\left(e_{2}, k_{2}\right)}^{r_{2} n_{2}}\right)^{*}=\left(\sigma_{\left(\operatorname{sgn}\left(e_{1} k_{1}+e_{2} k_{2}\right),\left|e_{1} k_{1}+e_{2} k_{2}\right|\right)}^{r_{1,2} n_{1,2}}\right)^{*} \\
=\sigma_{\left(-s g n\left(e_{1} k_{1}+e_{2} k_{2}\right),\left|e_{1} k_{1}+e_{2} k_{2}\right|\right)}^{\left(* r_{1,2}\right) n_{1,2}} \\
=\sigma_{\left(\operatorname{sgn}\left(-e_{1} k_{1}-e_{2} k_{2}\right),\left|-e_{1} k_{1}-e_{2} k_{2}\right|\right)}^{\left(* r_{1,2}\right) n_{1,2}}
\end{gathered}
$$

for some $r_{1,2} \in\{1, *\}$ and $n_{1,2} \in \mathbb{N}_{0}$

$$
\begin{gathered}
=\sigma_{\left(\operatorname{sgn}\left(-e_{2} k_{2}-e_{1} k_{1}\right),\left|-e_{2} k_{2}-e_{1} k_{1}\right|\right)}^{\left(* r_{2,1}\right) n_{2,1}}=\left(\sigma_{\left(-e_{2}, k_{2}\right)}^{\left(* r_{2}\right) n_{2}}\right)\left(\sigma_{\left(-e_{1}, k_{1}\right)}^{\left(* r_{1}\right) n_{1}}\right) \\
=\left(\sigma_{\left(e_{2}, k_{2}\right)}^{r_{2} n_{2}}\right)^{*}\left(\sigma_{\left(e_{1}, k_{1}\right)}^{r_{1} n_{1}}\right)^{*} .
\end{gathered}
$$

Thus, one can verify that, for any $T_{1}, T_{2} \in \mathcal{A}$,

$$
\left(T_{1} T_{2}\right)^{*}=T_{2}^{*} T_{1}^{*} \text { in } \mathcal{A},
$$

by (114) and (117).

Finally, for any $t \in \mathbb{C}$, and $\sigma_{(e, k)}^{r n} \in \sum(\mathbb{L} \mathbb{S})$,

$$
\left(t \sigma_{(e, k)}^{r n}\right)^{*}=\bar{t} \sigma_{(-e, k)}^{(* r) n}=\bar{t}\left(\sigma_{(e, k)}^{r n}\right)^{*},
$$

by (117), and, hence,

$$
(t T)^{*}=\bar{t} T^{*} \text { in } \mathcal{A}, \forall T \in \mathcal{A} \text {, and } t \in \mathbb{C},
$$

by (114) and (120).

Proposition 10. Let $\mathcal{A}$ be the pi-shift-operator algebra (114) generated by the pi-shift-operator semigroup $\sum(\mathbb{L S})$ in the operator space $B(\mathbb{L S})$, and let $(*)$ be the unary operation (117) on $\mathcal{A}$. Then, the operation ( $*$ ) is a well-defined adjoint on $\mathcal{A}$. Equivalently, every pi-shift operator $T \in \mathcal{A}$ is adjointable (in the sense of [2]) in $B(\mathbb{L} \mathbb{S}$ ).

Proof. The operation $(*)$ of $(117)$ is a well-defined adjoint on the pi-shift-operator algebra $\mathcal{A}$, by (118), (119), (120) and (121). Therefore, all elements of $\mathcal{A}$ are adjointable in $B(\mathbb{L S}$ ) (in the sense of [2]).

By the above proposition, one can understand the Banach algebra $\mathcal{A}$ is a well-determined Banach *-algebra embedded in $B(\mathbb{L} \mathbb{S})$. 


\section{Certain pi-Shift-Operators of $\mathcal{A}\left(\sum(\mathbb{L} \mathbb{S})\right)$}

Let $\mathcal{A} \stackrel{\text { denote }}{=} \mathcal{A}\left(\sum(\mathbb{L} S)\right)$ be the pi-shift-operator algebra (114) generated by the pi-shift-operator semigroup $\sum(\mathbb{L S})$ of $(109)$, embedded in the operator space $B(\mathbb{L S})$. By (112), each pi-shift operator $T \in \mathcal{S}$ has its expression,

$$
T=\sum_{(r, n,(e, k)) \in \mathbb{N}_{0}^{* \pm}} t_{(e, k)}^{r n} \sigma_{(e, k)}^{r n}, \text { with } t_{(e, k)}^{r n} \in \mathbb{C},
$$

in $\mathcal{A}$, where $\mathcal{S}$ is the subspace (112) in $\mathcal{A}$, and $\sum$ is the finite sum, where $\sigma_{(e, k)}^{r n} \in \sum(\mathbb{L} \mathbb{S})$, for all

$$
(r, n,(e, k)) \in \mathbb{N}_{0}^{* \pm}=\{1, *\} \times \mathbb{N}_{0} \times \mathbb{N}_{0}^{ \pm} .
$$

In this section, we concentrate on studying pi-shift operators $T \in \mathcal{S}$ having their forms (122) in the pi-shift-operator algebra $\mathcal{A}$.

Since $\mathcal{A}$ is a Banach $*$-algebra equipped with its adjoint (117), one can consider the following operator-theoretic properties on $\mathcal{A}$, classifying some types of pi-shift operators of $\mathcal{A}$, like in the usual Hilbert-space-operator spectral theory.

Definition 23. Let $T \in \mathcal{A}$ be a pi-shift operator on $\mathbb{L} \mathbb{S}$.

(i) $T$ is self-adjoint in $\mathcal{A}$, if $T^{*}=T$ on $\mathbb{L} \mathbb{S}$.

(ii) $T$ is a projection in $\mathcal{A}$, if $T^{*}=T=T^{2}$ on $\mathbb{L} \mathbb{S}$.

(iii) $T$ is normal in $\mathcal{A}$, if $T^{*} T=T T^{*}$ on $\mathbb{L} \mathbb{S}$.

(iv) $T$ is an isometry in $\mathcal{A}$, if $T^{*} T=1_{\mathbb{L S}}$ on $\mathbb{L} \mathbb{S}$.

(v) $T$ is a unitary in $\mathcal{A}$, if $T^{*} T=1_{\mathbb{L S}}=T T^{*}$ on $\mathbb{L} \mathbb{S}$.

Before studying the above operator-theoretic properties, we consider first that if $T \in \mathcal{S} \subset \mathcal{A}$ is a pi-shift operator (122), then it is decomposed by

$$
T=T_{*}+t_{0} 1_{\mathbb{L} S}+T_{1} i n \mathcal{A}, \text { for some } t_{0} \in \mathbb{C},
$$

with

$$
T_{*}=\sum_{(*, l,(r, m)) \in \mathbb{N}_{0}^{* \pm}} t_{(r, m)}^{* l} \sigma_{(r, m)^{\prime}}^{* l}
$$

and

$$
T_{1}=\sum_{(1, n,(e, k)) \in \mathbb{N}_{0}^{* \pm}} t_{(e, k)}^{1 n} \sigma_{(e, k)}^{1 n}
$$

Lemma 3. Let $T \in \mathcal{S} \subset \mathcal{A}$ be a pi-shift operator (122) with its decomposition $T_{*}+t_{0} 1_{\mathbb{L S}}+T_{1}$ of (123). Then,

$$
\begin{aligned}
& T_{*}^{*}=\sum_{(*, l,(r, m)) \in \mathbb{N}_{0}^{* \pm}} \overline{t_{(r, m)}^{* l}} \sigma_{(-r, m)^{\prime}}^{1 l} \\
& \text { and } \\
& T_{1}^{*}=\sum_{(1, n,(e, k)) \in \mathbb{N}_{0}^{* \pm}} \overline{\bar{t}_{(e, k)}^{1 n}} \sigma_{(-e, k)^{\prime}}^{* n}
\end{aligned}
$$

in $\mathcal{A}$. Thus, the adjoint $T^{*}$ of $T$ satisfies 


$$
\begin{aligned}
T^{*}= & \left(\sum_{(1, n,(e, k)) \in \mathbb{N}_{0}^{* \pm}} \overline{t_{(e, k)}^{1 n}} \sigma_{(-e, k)}^{* n}\right) \\
& +\overline{t_{0}} 1_{\mathbb{L S}}+\left(\sum_{(*, l,(r, m)) \in \mathbb{N}_{0}^{* \pm}} \overline{t_{(r, m)}^{* l}} \sigma_{(-r, m)}^{1 l}\right),
\end{aligned}
$$

in $\mathcal{A}$.

Proof. The proofs of (124) and (125) are done by (107).

Note in (124) and (125) that

$$
\begin{aligned}
& \sigma_{(e, k)}^{1 n} \in \sigma(\mathbb{L} \mathbb{S}) \subset \sum(\mathbb{L} \mathbb{S}) \stackrel{(*)}{\longmapsto} \sigma_{(-e, k)}^{* n} \in \sum(\mathbb{L} \mathbb{S}) \backslash \sigma(\mathbb{L} \mathbb{S}), \\
& \text { and } \\
& 0_{\mathbb{L} \mathbb{S}} \neq \sigma_{(e, k)}^{* n} \in \sum(\mathbb{L} \mathbb{S}) \backslash \sigma(\mathbb{L} \mathbb{S}) \stackrel{(*)}{\longmapsto} \sigma_{(-e, k)}^{1 n} \in \sigma(\mathbb{L} \mathbb{S}),
\end{aligned}
$$

on $\mathcal{A}$, by (117).

Thus, if a pi-shift operator $T$ is decomposed to be (123), then the adjoint $T^{*}$ of $T$ is decomposed to be

$$
T^{*}=\left(T^{*}\right)_{*}+\overline{t_{0}} 1_{\mathbb{L S}}+\left(T^{*}\right)_{1}
$$

with

$$
\left(T^{*}\right)_{*}=T_{1}^{*} \text {, and }\left(T^{*}\right)_{1}=T_{*}^{*}
$$

in $\mathcal{A}$.

Theorem 10. Let $T \in \mathcal{S} \subset \mathcal{A}$ be a pi-shift operator (122) decomposed to be $T_{*}+t_{0} 1_{\mathbb{L} \mathbb{S}}+T_{1}$, by (123). Then, $T$ is self-adjoint in $\mathcal{A}$, if and only if

$$
T_{1}^{*}=T_{*} \text { in } \mathcal{A} \text {, and } t_{0} \in \mathbb{R}
$$

Proof. Let $T=T_{*}+t_{0} 1_{\mathbb{L S}}+T_{1} \in \mathcal{S} \subset \mathcal{A}$. Then,

$$
\begin{aligned}
& T^{*}=T_{*}^{*}+\overline{t_{0}} 1_{\mathbb{L S}}+T_{1}^{*} \text { by }(107) \\
& \quad=T_{1}^{*}+\overline{t_{0}} 1_{\mathbb{L S}}+T_{*}^{*},
\end{aligned}
$$

in the sense of (123) by (126)', in $\mathcal{S} \subset \mathcal{A}$. Thus, one has

$$
T^{*}=T \text { in } \mathcal{A},
$$

if and only if

$$
T_{1}^{*}=T_{*}, T_{*}^{*}=T_{1} \text {, and } t_{0}=\overline{t_{0}} \text { in } \mathbb{C},
$$

if and only if

$$
T_{1}^{*}=T_{*} \text { in } \mathcal{A} \text {, and } t_{0} \in \mathbb{R} \text {. }
$$


Let $T \in \mathcal{S} \subset \mathcal{A}$ be a pi-shift operator (122). Then, one may write $T$ as follows:

$$
T=\left(\sum_{i=1}^{N_{*}} w_{l} \sigma_{\left(r_{i}, m_{i}\right)}^{* l_{i}}\right)+t_{0} 1_{\mathbb{L} \mathbb{S}}+\left(\sum_{s=1}^{N_{1}} t_{k} \sigma_{\left(e_{s}, k_{s}\right)}^{1 n_{s}}\right),
$$

with

$$
T_{*}=\sum_{i=1}^{N_{*}} w_{i} \sigma_{\left(r_{i}, m_{i}\right)}^{* l_{l}}, \text { and } T_{1}=\sum_{s=1}^{N_{1}} t_{s} \sigma_{\left(e_{s}, k_{s}\right)^{\prime}}^{1 n_{s_{s}}}
$$

in $\mathcal{A}$, by (123), for some $w_{i}, t_{s} \in \mathbb{C}$, for $i=1, \ldots, N_{*}$, and $s=1, \ldots, N_{1}$, for $N_{*}, N_{1} \in \mathbb{N}$.

Let $T \in \mathcal{S} \subset \mathcal{A}$ be a pi-shift operator (128). Then, it is self-adjoint in $\mathcal{A}$, if and only if $t_{0} \in \mathbb{R}$, and $T_{*}=T_{1}^{*}$ in $\mathcal{A}$, by (127), if and only if $t_{0} \in \mathbb{R}$, and

$$
\begin{aligned}
& N_{1}=N_{*} i n \mathbb{N}, \\
& \text { and } \\
& w_{i}=\bar{t}_{i} \text { in } \mathbb{C}, \forall i=1, \ldots, N_{1}=N_{*}, \\
& \text { and } \\
& l_{i}=n_{i} i n \mathbb{N}_{0}, \forall i=1, \ldots, N_{1},
\end{aligned}
$$

and

$$
\left(r_{i}, m_{i}\right)=\left(-e_{i}, k_{i}\right) \text { in } \mathbb{N}_{0}^{ \pm}, \forall i=1, \ldots, N_{1},
$$

by (128).

Corollary 3. Let $T \in \mathcal{S} \subset \mathcal{A}$ be a pi-shift operator (128). Then, $T$ is self-adjoint in $\mathcal{A}$, if and only if

$$
\begin{aligned}
& t_{0} \in \mathbb{R}, \text { and } N_{*}=N_{1} i n \mathbb{N}, \\
& \text { and } \\
& w_{i}=\overline{t_{i}}, \text { foralli }=1, \ldots, N_{1}, \\
& \text { and } \\
& \left(l_{i},\left(r_{i}, m_{i}\right)\right)=\left(n_{i},\left(-e_{i}, k_{i}\right)\right) \operatorname{in} \mathbb{N}_{0} \times \mathbb{N}_{0}^{ \pm},
\end{aligned}
$$

for all $i=1, \ldots, N_{1}$.

Proof. The characterization (130) of the self-adjointness of a pi-shift operator (128) is obtained by the self-adjoint characterization (127) and (129).

Example 1. Let $T_{1}, T_{2}, T_{3} \in \mathcal{S} \subset \mathcal{A}$ be pi-shift operators as follows:

$$
\begin{gathered}
T_{1}=i \sigma_{(-, 1)}^{* 2}+3 \cdot 1_{\mathbb{L S}}-i \sigma_{(+, 1)^{\prime}}^{2} \\
T_{2}=\sigma_{(-, 2)}^{* 3}+2 i \sigma_{(-, 3)}^{* 1}-2 i \sigma_{(+, 3)}^{1}+\sigma_{(+, 2)}^{3} \text { and } \\
T_{3}=3 \sigma_{(-, 3)}^{* 5}-2 \sigma_{(-, 2)}^{* 3}+i \sigma_{(-, 1)}^{* 1}+\sqrt{2} \cdot 1_{\mathbb{L S}}-i \sigma_{(+, 1)}^{1}-2 \sigma_{(+, 2)}^{3}+3 \sigma_{(+, 3)}^{5} .
\end{gathered}
$$

Then, they are self-adjoint in $\mathcal{A}$, by (127) and (130).

Let's consider general cases.

Theorem 11. Let $U \in \mathcal{A}$ be an arbitrary pi-shift operator. Then, the operators

$$
T_{1}=t_{1} U U^{*}, \quad T_{2}=t_{2} U^{*} U,
$$


and

$$
T_{3}=t_{3}\left(U+U^{*}\right)=t_{3}\left(U^{*}+U\right),
$$

for $t_{1}, t_{2}, t_{3} \in \mathbb{R}$, are self-adjoint in $\mathcal{A}$.

Moreover, if $U_{1}$ and $U_{2}$ are self-adjoint pi-shift operators of $\mathcal{A}$, then $s_{1} U_{1}+s_{2} U_{2}$ is self-adjoint in $\mathcal{A}$, too, for all $s_{1}, s_{2} \in \mathbb{R}$ in $\mathbb{C}$.

Proof. Let $U \in \mathcal{A}$ be an arbitrary pi-shift operator (including the case where $U \in \mathcal{A} \backslash \mathcal{S}$ ), and let $t_{1}, t_{2}, t_{3}$ $\in \mathbb{R}$. Then, the pi-shift operators $T_{1}, T_{2}$ and $T_{3}$ satisfy that

$$
\begin{aligned}
& T_{1}^{*}=\left(t_{1} U U^{*}\right)^{*}=\overline{t_{1}} U^{* *} U^{*}=t_{1} U U^{*}, \\
& T_{2}^{*}=\left(t_{2} U^{*} U\right)^{*}=\overline{t_{2}} U^{*} U^{* *}=t_{2} U^{*} U,
\end{aligned}
$$

and

$$
\begin{aligned}
T_{3}^{*} & =\left(t_{3}\left(U+U^{*}\right)\right)^{*} \\
& =\overline{t_{3}}\left(U^{*}+U^{* *}\right) \\
& =t_{3}\left(U+U^{*}\right)=T_{3},
\end{aligned}
$$

in $\mathcal{A}$. Therefore, they are self-adjoint in $\mathcal{A}$.

Now, assume that $U_{1}$ and $U_{2}$ are self-adjoint in $\mathcal{A}$, and let $s_{1}, s_{2} \in \mathbb{R}$. If

$$
T=s_{1} U_{1}+s_{2} U_{2} \in \mathcal{A},
$$

then

$$
T^{*}=\overline{s_{1}} U_{1}^{*}+\overline{s_{2}} U_{2}^{*}=s_{1} U_{1}+s_{2} U_{2}=T,
$$

in $\mathcal{A}$, and hence, it is self-adjoint in $\mathcal{A}$.

Now, let's focus on pi-shift operators of $\mathcal{A}$, contained in the subspace $\mathcal{S}$ of (112) in $B(\mathbb{L S})$. Let $T \in \mathcal{S}$ $\subset \mathcal{A}$ be a pi-shift operator (128) with its decomposition (123). Such an operator $T$ is self-adjoint in $\mathcal{A}$, if and only if

$$
T=T_{1}^{*}+t_{0} 1_{\mathbb{L S}}+T_{1} \text { with } t_{0} \in \mathbb{R},
$$

by (127). Observe first that

$$
\begin{aligned}
T^{2} & =\left(T_{1}^{*}+t_{0} 1_{\mathbb{L S}}+T_{1}\right)\left(T_{1}^{*}+t_{0} 1_{\mathbb{L S}}+T_{1}\right) \\
& =\left(T_{1}^{*}\right)^{2}+2 t_{0} T_{1}^{*}+T_{1}^{*} T_{1}+t_{0}^{2} 1_{\mathbb{L S}}+T_{1} T_{1}^{*}+2 t_{0} T_{1}+T_{1}^{2} .
\end{aligned}
$$

In the above computation (131), one can realize immediately that, if $T_{1}=\sigma_{(e, k)}^{n}$ with $n \neq 0$, then

$$
T^{2} \neq T \text { in } \mathcal{A} \text {. }
$$

This observation (132) motivates us to verify the following result.

Lemma 4. Let $T \in \mathcal{S} \subset \mathcal{A}$ be a non-zero, self-adjoint, finite-sum pi-shift operator,

$T=\left(\sum_{s=1}^{N} \overline{t_{s}} \sigma_{\left(-e_{s}, k_{s}\right)}^{* n_{s}}\right)+t_{0} 1_{\mathbb{L S}}+\left(\sum_{s=1}^{N} t_{s} \sigma_{\left(e_{s}, k_{s}\right)}^{1 n_{s}}\right)$, for $N \in \mathbb{N}$. If $t_{N} \neq 0$ in $\mathbb{C}$, then $T$ is not a projection in $\mathcal{A}$. The converse also holds true too. 
Proof. Suppose $T$ is given as above. Then, by (127) and (130), this operator $T$ is self-adjoint in $\mathcal{A}$. Thus, to check the projection-property for such $T$, it suffices to check $T^{2}=T$ in $\mathcal{A}$.

However, if $t_{N} \neq 0$ in $\mathbb{C}$, for $N \in \mathbb{N}$, then the pi-shift operator $T^{2}$ contains non-zero summands $\left(\overline{t_{N}}\right)^{2} \sigma_{\left(-e_{N}, 2 k_{N}\right)}^{*\left(2 n_{N}\right)}$ and $t_{N}^{2} \sigma_{\left(e_{N}, 2 k_{N}\right)}^{2 n_{N}}$ in $\mathcal{A}$, by (131), i.e., $T^{2} \neq T$ in $\mathcal{A}$. Thus, if $t_{N} \neq 0$ in $\mathbb{C}$, for $N \in \mathbb{N}$, then $T$ cannot be a projection in $\mathcal{A}$.

Conversely, suppose $T$ is a projection in $\mathcal{A}$. Then, $T^{2}=T$ in $\mathcal{A}$. If $t_{N} \neq 0$ in $\mathbb{C}$, for $N \in \mathbb{N}$, then $T^{2} \neq T$ in $\mathcal{A}$, by (131). It contradicts our assumption that $T$ is a projection in $\mathcal{A}$.

The above lemma shows that if a non-zero, self-adjoint, finite-sum pi-shift operator is self-adjoint, then $T$ cannot be a projection in $\mathcal{A}$ in the sense of (ii) in Definition 23.

Theorem 12. Let $T \in \mathcal{S} \subset \mathcal{A}$ be a finite-sum pi-shift operator (130). Then, $T$ is a projection, if and only if

$$
T=0_{\mathbb{L S}}, \text { or } 1_{\mathbb{L S}} \text { in } \mathcal{A} \text {. }
$$

Proof. By the above lemma, a given self-adjoint finite-sum pi-shift operator $T$ is a projection, if and only if

$$
T=t_{0} 1_{\mathbb{L S}}, \text { for some } t_{0} \in \mathbb{R} .
$$

Then, such a pi-shift operator $T$ is a projection in $\mathcal{A}$, if and only if

$$
T^{2}=t_{0}^{2} 1_{\mathbb{L S}}=t_{0} 1_{\mathbb{L S}}=T \text { in } \mathcal{A},
$$

if and only if

$$
t_{0}^{2}=t_{0} \text { in } \mathbb{R} \Longleftrightarrow t_{0}^{2}-t_{0}=0 \text { in } \mathbb{R}
$$

if and only if

$$
t_{0}=0 \text {, or } t_{0}=1 \text { in } \mathbb{R}
$$

if and only if

$T=0 \cdot 1_{\mathbb{L S}}=0_{\mathbb{L S}}$, or $T=1_{\mathbb{L S}}$, in $\mathcal{A}$.

Therefore, the projection-property (133) holds for $T \in \mathcal{S}$ in $\mathcal{A}$.

The above theorem illustrates that the only "finite-sum" projections of $\mathcal{S}$ are either $0_{\mathbb{L S}}$ or $1_{\mathbb{L S}}$ in $\mathcal{A}$. Meanwhile, one can obtain the following projection-property on $\mathcal{A} \backslash \mathcal{S}$ too.

Theorem 13. Let $\sigma=\sigma_{(e, k)}^{n} \in \sigma(\mathbb{L} \mathbb{S}) \subset \sum(\mathbb{L} \mathbb{S})$ in $\mathcal{A}$, for $n \in \mathbb{N}_{0},(e, k) \in \mathbb{N}_{0}^{ \pm}$. Then, the pi-shift operators $P_{1}=$ $\sigma^{*} \sigma$, and $P_{2}=\sigma \sigma^{*}$ are projections in $\mathcal{A}$ in the sense of (ii) in Definition 23.

Proof. Let $P_{1}$ and $P_{2}$ be given as above. Note that $P_{1}=\sigma^{*} \sigma=1_{\mathbb{L S}}$ in $\mathcal{A}$. Indeed,

$$
\begin{aligned}
\sigma^{*} \sigma & =\sigma_{(-e, k)}^{* n} \sigma_{(e, k)}^{n}=G^{* n} \beta_{-e}^{k} G^{n} \beta_{e}^{k} \\
& =G^{* n} G^{n} \beta_{-e}^{k} \beta_{e}^{k}=\left(G^{* n} G^{n}\right) \beta_{s g n(-e k+e k)}^{\mid-e k+e k} \\
& =\left(1_{\mathbb{L} \mathbb{S}}\right)\left(1_{\mathbb{L} \mathbb{S}}\right)=1_{\mathbb{L} \mathbb{S}} .
\end{aligned}
$$

Thus, it satisfies the projection-property, by (133).

Observe now that

$$
P_{2}^{*}=\left(\sigma \sigma^{*}\right)^{*}=\sigma^{* *} \sigma^{*}=\sigma \sigma^{*}=P_{2},
$$

in $\mathcal{A}$, and, hence, it is self-adjoint in $\mathcal{A}$. Moreover, 


$$
\begin{aligned}
P_{2}^{2} & =\left(\sigma \sigma^{*}\right)\left(\sigma \sigma^{*}\right)=\sigma\left(\sigma^{*} \sigma\right) \sigma^{*} \\
& =\sigma\left(1_{\mathbb{L S}}\right) \sigma^{*}=\sigma \sigma^{*}=P_{2} .
\end{aligned}
$$

Therefore, the self-adjoint pi-shift operator $P_{2}$ is a projection in $\mathcal{A}$ in the sense of (ii) in Definition 23.

The above two theorems show that: (i) the finite-sum pi-shift operator $T \in \mathcal{S}$ is a projection in $\mathcal{A}$, if and only if either $T=0_{\mathbb{L S}}$, or $T=1_{\mathbb{L} \mathbb{S}}$; and the pi-shift operators formed by $\sigma^{*} \sigma$ and $\sigma \sigma^{*}$ are projections in $\mathcal{A}$, for all generating operators $\sigma \in \sum(\mathbb{L} \mathbb{S})$ of the pi-shift-operator algebra $\mathcal{A}$.

Now, let $\sigma=\sigma_{(e, k)}^{n} \in \sigma(\mathbb{L} \mathbb{S}) \subset \sum(\mathbb{L} \mathbb{S})$ in the pi-shift-operator algebra $\mathcal{A}$. Assume that $n \neq 0$ in $\mathbb{N}_{0}$. Then, it is not hard to show that $\sigma^{*} \sigma \neq \sigma \sigma^{*}$ on $\mathbb{L} \mathbb{S}$. Indeed, if $p$ is an $n_{p}$-th prime in the TOset $\mathcal{P}$ of (67), for some $n_{p} \in \mathbb{N}$, and if $n \geq n_{p}$ in $\mathbb{N}$, then

$$
\begin{aligned}
& \sigma^{*} \sigma\left(\Theta_{p, j}\right)=\Theta_{p, j}=1_{\mathbb{L S}}\left(\Theta_{p, j}\right), \\
& \text { but } \\
& \sigma \sigma^{*}\left(\Theta_{p, j}\right)=\sigma\left(0_{\mathbb{L S}}\right)=0_{\mathbb{L} \mathbb{S},}
\end{aligned}
$$

for the corresponding semicircular element $\Theta_{p, j} \in \mathcal{X}$.

The above relation (134) directly demonstrates that our pi-shift operators are not normal in $\mathcal{A}$ in the sense of (iii) in Definition 23, in general. Remark that, if $n=0$ in $\mathbb{N}_{0}$, then

$$
\sigma=\sigma_{(e, k)}^{n}=\sigma_{(e, k)}^{0}=G^{0} \beta_{e}^{k}=1_{\mathbb{L S}} \cdot \beta_{e}^{k}=\beta_{e}^{k}
$$

Thus, in such a case, this pi-shift operator $\sigma$ can be normal in $\mathcal{A}$, because

$$
\begin{aligned}
\left(\beta_{e}^{k}\right)^{*} \beta_{e}^{k} & =\beta_{-e}^{k} \beta_{e}^{k}=\beta_{s g n(-e k+e k)}^{\mid-e k+e k} \\
& =\beta_{ \pm}^{0}=1_{\mathbb{L S}}=\beta_{e}^{k} \beta_{-e}^{k}=\beta_{e}^{k}\left(\beta_{e}^{k}\right)^{*},
\end{aligned}
$$

by (107). Thus, if $n=0$ in $\mathbb{N}_{0}$, then

$$
\text { for all }(e, k) \in \mathbb{N}_{0}^{ \pm} . \quad\left(\sigma_{(e, k)}^{0}\right)^{*} \sigma_{(e, k)}^{0}=1_{\mathbb{L S}}=\sigma_{(e, k)}^{0}\left(\sigma_{(e, k)}^{0}\right)^{*},
$$

Theorem 14. Let $\sigma^{n} \stackrel{\text { denote }}{=} \sigma_{(e, k)}^{n} \in \sigma(\mathbb{L S}) \subset \sum(\mathbb{L S})$ in the pi-shift-operator algebra $\mathcal{A}$, for $n \in \mathbb{N}_{0},(e, k) \in \mathbb{N}_{0}^{ \pm}$.

(i) $\sigma^{n}$ is normal in $\mathcal{A}$ in the sense of (iii) in Definition 23, if and only if $n=0$ in $\mathbb{N}_{0}$.

(ii) $\sigma^{0}$ is a unitary in $\mathcal{A}$ in the sense of (v) in Definition 23.

(iii) $\sigma^{n}$ is a unitary in $\mathcal{A}$ in the sense of (v) in Definition 23, if and only if $n=0$ in $\mathbb{N}_{0}$.

(iv) $\sigma^{0}$, and $\left(\sigma^{0}\right)^{*}$ are isometries in $\mathcal{A}$ in the sense of (iv) in Definition 23.

Proof. Let $\sigma^{n} \in \sigma(\mathbb{L} \mathbb{S}) \subset \mathcal{A}$ be given as above. By (135), if $n=0$ in $\mathbb{N}_{0}$, then $\sigma^{n}$ is normal in $\mathcal{A}$. Suppose now $\sigma^{n}$ is normal, where $n \neq 0$ in $\mathbb{N}_{0}$. Since $n \neq 0$, one has

$$
\left(\sigma^{n}\right)^{*} \sigma^{n} \neq \sigma^{n}\left(\sigma^{n}\right)^{*} \text { on } \mathbb{L} \mathbb{S},
$$

by (134). Equivalently, $\sigma^{n}$ is not normal in $\mathcal{A}$. It contradicts our assumption that it is normal. Therefore, the statement $(i)$ in Theorem 14 holds.

The relation (135) shows not only that $\sigma^{0}$ is normal, but also it is unitary in $\mathcal{A}$. Thus, the statement (ii) in Theorem 14 is shown.

By using the similar arguments of the proof of (i) in Theorem 14, the statement (iii) in Theorem 14 holds by (ii) in Theorem 14 . 
The statement (iv) in Theorem 14 is proven by (ii) and (iii) in Theorem 14 and the isometry-property (iv) in Definition 23 in $\mathcal{A}$.

Now, let's focus on the normality (iii) in Definition 23 for pi-shift operators $T \in \mathcal{S}$ in $\mathcal{A}$. Let $T \in \mathcal{S}$ be a finite-sum pi-shift operator, decomposed to be

$$
T=T_{*}+t_{0} 1_{\mathbb{L S}}+T_{1}, \text { as in (123). }
$$

By (i) in Theorem 14, one can easily verify that $T$ is not normal in $\mathcal{A}$, in general.

Lemma 5. Let $T \in \mathcal{S}$ be a pi-shift operator,

$$
T=\sum_{(r, 0,(e, k)) \in \mathbb{N}_{0}^{* \pm}} t_{(e, k)}^{r 0} \sigma_{(e, k)}^{r 0} \in \mathcal{A}
$$

Then, it is normal in $\mathcal{A}$ in the sense of (iii) in Definition 23.

Proof. As we discussed in (135),

$$
\sigma_{(e, k)}^{r 0} \stackrel{\text { def }}{=} G^{r 0} \beta_{e}^{k}=\beta_{e}^{k}
$$

by (105) and (109), for all $(r, 0,(e, k)) \in \mathbb{N}_{0}^{* \pm}$. Thus, the given pi-shift operator $T$ of (136) can be re-written by

$$
T=\sum_{(r, 0,(e, k)) \in \mathbb{N}_{0}^{* \pm}} t_{(e, k)}^{r 0} \sigma_{(e, k)}^{r 0}=\sum_{(e, k) \in \mathbb{N}_{0}^{ \pm}} s_{(e, k)} \beta_{e}^{k},
$$

in $\mathcal{S} \subset \mathcal{A}$, where the coefficients $s_{(e, k)} \in \mathbb{C}$ are determined uniquely to be

$$
s_{(e, k)}=t_{(-, k)}^{* 0}+t_{(+, k)}^{10} \text { for all }(e, k) \in \mathbb{N}_{0}^{ \pm} \backslash\{( \pm, 0)\}
$$

and

$$
s_{(e, 0)}=t_{(e, 0)}, \text { for all } e \in\{ \pm\} .
$$

If we understand the pi-shift operator $T$ of (136) as (137), then,

$$
T^{*}=\sum_{(e, k) \in \mathbb{N}_{0}^{ \pm}} \overline{s_{(e, k)}} \beta_{-e}^{k} \in \mathcal{A}
$$

by (107) and (137).

Observe now that

$$
\begin{aligned}
T^{*} T & =\sum_{\left(\left(e^{\prime}, k^{\prime}\right),(e, k)\right) \in \mathbb{N}_{0}^{ \pm} \times \mathbb{N}_{0}^{\times}} \overline{\bar{s}\left(e^{\prime}, k^{\prime}\right)} s_{(e, k)} \beta_{-e^{\prime}}^{k^{\prime}} \beta_{e}^{k} \\
& =\sum_{\left(\left(e^{\prime}, k^{\prime}\right),(e, k)\right) \in \mathbb{N}_{0}^{ \pm} \times \mathbb{N}_{0}^{ \pm}}\left(\overline{s_{\left(e^{\prime}, k^{\prime}\right)}} s_{(e, k)}\right) \beta_{s g n\left(-e^{\prime} k^{\prime}+e k\right)}^{\left|-e^{\prime} k^{\prime} e k\right|} \\
& =\sum_{\left(\left(e^{\prime}, k^{\prime}\right),(e, k)\right) \in \mathbb{N}_{0}^{ \pm} \times \mathbb{N}_{0}^{ \pm}}\left(s_{(e, k)} \overline{s\left(e^{\prime}, k^{\prime}\right)}\right) \beta_{s g n\left(e k-e^{\prime} k^{\prime}\right)}^{\left|e k-k^{\prime}\right|} \\
& =\sum_{\left((e, k),\left(e^{\prime}, k^{\prime}\right)\right) \in \mathbb{N}_{0}^{ \pm} \times \mathbb{N}_{0}^{ \pm}}\left(s_{(e, k)} \overline{s\left(e^{\prime}, k^{\prime}\right)}\right) \beta_{e}^{k} \beta_{-e^{\prime}}^{k^{\prime}}=T T^{*},
\end{aligned}
$$

in $\mathcal{A}$. Therefore, this pi-shift operator $T$ is normal in $\mathcal{A}$, by (138). 
The above lemma shows that pi-shift operators $T \in \mathcal{S}$ formed by (136) are normal in $\mathcal{A}$, in the sense of (iii) in Definition 23. How about the converse of this lemma for the elements of $\mathcal{S}$ in $\mathcal{A}$ ? One can verify that the converse does not hold in general. Indeed, from the self-adjoint characterization (127), there are normal pi-shift operators which are not of the forms (136) because the self-adjointness (i) in Definition 23 implies the normality (iii) in Definition 23. Generally, one can get the following result.

Lemma 6. There are normal pi-shift operators in the sense of (iii) in Definition 23 in $\mathcal{S} \subset \mathcal{A}$, which are not formed by (136). For example, the converse of the above lemma does not hold true.

Proof. First, note that, if a pi-shift operator $T$ is self-adjoint in $\mathcal{A}$ in the sense of $(i)$ in Definition 23 , then it is normal in $\mathcal{A}$, by definition. Thus, it is proven by construction. By (127) and (130), if

$$
T=\sigma_{(-, 3)}^{* 2}+\sigma_{(+, 3)}^{2} \in \mathcal{A} \text {. }
$$

Then, it is self-adjoint, and, hence, normal in $\mathcal{A}$. Clearly, $T$ is not of the form (136) in $\mathcal{S} \subset \mathcal{A}$.

Then, how about the following restricted case? We will restrict our interests to the cases where a "normal" pi-shift operator $T \in \mathcal{S}$ is "not self-adjoint" in $\mathcal{A}$.

Lemma 7. Let $T \in \mathcal{S} \subset \mathcal{A}$ be a "non-zero" pi-shift operator (122), and suppose $T$ is "not" self-adjoint in $\mathcal{A}$. If $T$ is normal in $\mathcal{A}$, then $T$ is formed by (136).

Proof. Suppose either $T=\sigma_{(e, k)}^{n}$, or $\sigma_{(e, k)}^{* n}$ in $\mathcal{A}$, for some $n \in \mathbb{N}_{0},(e, k) \in \mathbb{N}_{0}^{ \pm}$. Then, such a finite-sum pi-shift operator $T$ is not normal in general, by $(i)$ in Theorem 14 .

Assume that $T$ contains at least one non-zero summand $s_{0} \sigma_{\left(e_{0}, k_{0}\right)}^{n_{0}}$, for some $n_{0} \in \mathbb{N},\left(e_{0}, k_{0}\right) \in \mathbb{N}_{0}^{ \pm}$, with $s_{0} \neq 0$ in $\mathbb{C}$, and $r \in\{1, *\}$. In addition, assume that $T$ does not contain a summand $\overline{s_{0}} \sigma_{\left(-e, k_{0}\right)}^{* n_{0}}$ in (122). One can take such a summand of $T$, since $T \neq 0_{\mathbb{L} S}$ is assumed not to be self-adjoint, by (127). For example, $T_{1}^{*}$ $\neq T_{*}$ in $\mathcal{A}$, in the sense of (123).

Then, by (134) and (i) in Theorem 14, the operator $T$ cannot be normal in $\mathcal{A}$, i.e., if $T \in \mathcal{S}$ is a non-zero, non-self-adjoint pi-shift operator satisfying the above condition, then

$$
T^{*} T \neq T T^{*} \text { on } \mathbb{L} \mathbb{S} \text {. }
$$

Conversely, let $T \in \mathcal{S} \subset \mathcal{A}$ be a non-zero, non-self-adjoint operator, and assume that $T$ is not formed by (136). Then, it is not normal in $\mathcal{A}$. Equivalently, if a non-zero, non-self-adjoint pi-shift operator $T \in \mathcal{S}$ is normal in $\mathcal{A}$, then it is formed by (136).

By the above three lemmas, we obtain the following normality condition in the pi-shift-operator algebra $\mathcal{A}$.

Theorem 15. Let $T \in \mathcal{S} \subset \mathcal{A}$ be a non-zero, non-self-adjoint pi-shift operator (122). Then, $T$ is normal, if and only if

$$
T=\sum_{(r, 0,(e, k))} t_{(e, k)}^{r 0} \sigma_{(e, k)}^{r 0}=\sum_{(e, k) \in \mathbb{N}_{0}^{ \pm}} s_{(e, k)} \beta_{e}^{k} o n \mathbb{L} \mathbb{S},
$$

with

$$
s_{(e, k)}=t_{(e, k)}^{* 0}+t_{(e, k)}^{1,0} \in \mathbb{C}, \text { for }(e, k) \in \mathbb{N}_{0}^{ \pm} \backslash\{( \pm, 0)\},
$$

and

$$
s_{(e, 0)}=t_{(e, 0)} \in \mathbb{C}, \text { for } e \in\{ \pm\}
$$


Proof. Note that $T \in \mathcal{S}$ is assumed to be non-zero, and non-self-adjoint in $\mathcal{A}$.

$(\Leftarrow)$ If $T$ is in the sense of (139), then it is normal in $\mathcal{A}$ by (138).

$(\Rightarrow)$ If $T$ is not formed by (139), then $T$ is not normal under conditions, by the very above lemma.

The above condition (139) characterizes the normality (iii) in Definition 23 of non-zero, non-self-adjoint, finite-sum pi-shift operators of $\mathcal{S}$ in $\mathcal{A}$.

By the unitarity ( $v$ ) in Definition 23 in the pi-shift-operator algebra $\mathcal{A}$, if a pi-shift operator $T$ is unitary, then it must be normal in $\mathcal{A}$ in the sense of (iii) in Definition 23. Thus, we restrict our interests to the unitarity $(v)$ in Definition 23 for normal pi-shift operators. Furthermore, let's assume that $T$ is normal, but "not" self-adjoint.

For example, to consider the pure-unitarity for $(v)$ in Definition 23, we restrict our interests to non-self-adjoint, normal pi-shift operators in $\mathcal{A}$. By (139), a pi-shift operator $T \in \mathcal{A}$ is normal, if and only if

$$
T=\sum_{(e, k) \in \mathbb{N}_{0}^{ \pm}} s_{(e, k)} \beta_{e}^{k} \in \mathcal{A} \text {, with } s_{(e, k)} \in \mathbb{C},
$$

where $\beta_{e}^{k}$ are the $k$-(e)-shifts on $\mathbb{L} \mathbb{S}$, for all $(e, k) \in \mathbb{N}_{0}^{ \pm}$.

Recall that, by (138), if $T$ is a normal operator (139), then

$$
T^{*} T=T T^{*}=\sum_{\left(\left(e^{\prime}, k^{\prime}\right),(e, k)\right) \in \mathbb{N}_{0}^{ \pm} \times \mathbb{N}_{0}^{ \pm}}\left(\overline{S_{\left(e^{\prime}, k^{\prime}\right)}} S_{(e, k)}\right) \beta_{s g n\left(e k-e^{\prime} k^{\prime}\right)^{\prime}}^{\mid e k-e^{\prime} k^{\prime}}
$$

in $\mathcal{A}$.

Thus, such a pi-shift operator $T \in \mathcal{S}$ is unitary in $\mathcal{A}$ in the sense of $(v)$ in Definition 23, if and only if

$$
\sum_{\left(\left(e^{\prime}, k^{\prime}\right),(e, k)\right) \in \mathbb{N}_{0}^{ \pm} \times \mathbb{N}_{0}^{ \pm}}\left(\overline{S_{\left(e^{\prime}, k^{\prime}\right)}}(e, k)\right) \beta_{s g n\left(e k-e^{\prime} k^{\prime}\right)}^{\mid e k-e^{\prime} k^{\prime}}=1_{\mathbb{L} \mathbb{S}} .
$$

Theorem 16. Let $T \in \mathcal{S} \subset \mathcal{A}$ be a normal pi-shift operator (139). Then, $T$ is unitary in $\mathcal{A}$, if and only if

$$
\begin{aligned}
& \sum_{(e, k) \in \mathbb{N}_{0}^{ \pm}}\left(\overline{s_{(-e, k)}} \mathcal{S}_{(e, k)}\right)=1, \\
& \text { and } \\
& \sum_{\left(\left(e^{\prime}, k^{\prime}\right),(e, k)\right) \in \mathbb{N}_{0}^{ \pm} \times \mathbb{N}_{0}^{ \pm}, e^{\prime} \neq-e \text { in }\{ \pm\}}\left(\overline{s_{\left(e^{\prime}, k^{\prime}\right)}}(e, k)\right)=0,
\end{aligned}
$$

in $\mathbb{C}$.

Proof. $(\Leftarrow)$ Suppose the condition $(140)$ holds for a given normal pi-shift operator $T$ of $(139)$. Then, by (138),

$$
\begin{gathered}
T^{*} T=T T^{*} \\
=\left(\sum_{(e, k) \in \mathbb{N}_{0}^{ \pm}}\left(\overline{S_{(-e, k)}}(e, k)\right)\right) \cdot 1_{\mathbb{L S}} \\
+\sum_{\left(\left(e^{\prime}, k^{\prime}\right),(e, k)\right) \in \mathbb{N}_{0}^{ \pm} \times \mathbb{N}_{0}^{ \pm}, e^{\prime} \neq-e \text { in }\{ \pm\}}\left(\overline{S_{\left(e^{\prime}, k^{\prime}\right)}} s_{(e, k)}\right) \beta_{s g n\left(e k-e^{\prime} k^{\prime}\right)}^{\left|e k-k^{\prime}\right|} \\
=1_{\mathbb{L S}}+0_{\mathbb{L S}}=1_{\mathbb{L S} S}
\end{gathered}
$$


Therefore, $T$ is unitary in $\mathcal{A}$ in the sense of ( $v$ ) in Definition 23.

$(\Rightarrow)$ Suppose that $T$ is unitary in $\mathcal{A}$, and assume that the condition (140) does not hold for $T$. Then, by (138),

$$
T^{*} T=T T^{*} \neq 1_{\mathbb{L S}} \text { in } \mathcal{A} .
$$

It contradicts our assumption that $T$ is unitary.

\section{Distorted Free Probability on $\mathbb{L S}$ by $\mathcal{A}\left(\sum(\mathbb{L} \mathbb{S})\right)$}

Let $\mathbb{L} \mathbb{S}$ be the semicircular Adelic filterization, and let $\mathcal{A} \stackrel{\text { denote }}{=} \mathcal{A}\left(\sum(\mathbb{L} \mathbb{S})\right)$ be the pi-shift-operator algebra in the operator space $B(\mathbb{L S})$. In Section 13 , we considered operator-theoretic properties on $\mathcal{A}$. Here, we study how such operators distort the original free-distributional data on $\mathbb{L} \mathbb{S}$.

Recall first that the subset $\sigma(\mathbb{L S})$ (which is the pi-shift monoid) of the pi-shift-operator semigroup $\sum(\mathbb{L} \mathbb{S})$ (generating $\mathcal{A}$ ) are consisting of injective free-homomorphisms contained in the homomorphism semigroup $\operatorname{Hom}(\mathbb{L} \mathbb{S})$, by (99). Thus, "some" generating operators of $\mathcal{A}$,

$$
\sigma_{(e, k)}^{1 n}=\sigma_{(e, k)}^{n} \in \sigma(\mathbb{L} \mathbb{S}) \subset \sum(\mathbb{L} \mathbb{S})
$$

preserve the free probability on $\mathbb{L} \mathbb{S}$. However, not "all" generators preserve the free probability on $\mathbb{L} \mathbb{S}$. For instance, a generating operator

$$
\sigma_{(e, k)}^{* n} \in \sum(\mathbb{L} \mathbb{S}) \backslash \sigma(\mathbb{L} S)
$$

is a $*$-homomorphism, but not a free-homomorphism on $\mathbb{L} \mathbb{S}$, whenever $n \neq 0$ in $\mathbb{N}_{0}$, for any $(e, k) \in$ $\mathbb{N}_{0}^{ \pm}$. Thus, it is interesting to check how the pi-shift-operator semigroup $\sum(\mathbb{L} \mathbb{S})$ deform the original free-distributional data on $\mathbb{L} \mathbb{S}$.

Lemma 8. Let $t \sigma_{(e, k)}^{1 n}=t \sigma_{(e, k)}^{n}, t \sigma_{(e, k)}^{* n} \in \mathcal{A}$ be pi-shift operators for $t \in \mathbb{C}$, where

$$
\sigma_{(e, k)}^{n} \in \sigma(\mathbb{L} \mathbb{S}) \subset \sum(\mathbb{L} \mathbb{S}), \text { in } \mathcal{A}
$$

and

$$
\sigma_{(e, k)}^{* n} \in \sum(\mathbb{L} \mathbb{S}) \backslash \sigma(\mathbb{L} \mathbb{S}), \text { in } \mathcal{A} .
$$

If $\Theta_{p, j} \in \mathcal{X}$ is a semicircular element in $\mathbb{L} \mathbb{S}$, then

$$
\begin{aligned}
& \tau^{0}\left(\left(t \sigma_{(e, k)}^{n}\left(\Theta_{p, j}\right)\right)^{l}\right)=t^{l}\left(\omega_{l} c_{\frac{l}{2}}\right), \\
& \text { and } \\
& \tau^{0}\left(\left(t \sigma_{(e, k)}^{* n}\left(\Theta_{p, j}\right)\right)^{l}\right)= \begin{cases}t^{l}\left(\omega_{l} c_{\frac{l}{2}}\right) & \text { if } \exists q \in \mathcal{P}, \text { s.t., } \\
g^{n}(q)=p \text { in } \mathcal{P} \\
0 & \text { otherwise, }\end{cases}
\end{aligned}
$$

for all $l \in \mathbb{N}$.

Proof. For convenience, let's denote

$$
\sigma \stackrel{\text { denote }}{=} \sigma_{(e, k)}^{n} \text {, and } \sigma^{*} \stackrel{\text { denote }}{=} \sigma_{(e, k)^{\prime}}^{* n}
$$

in the pi-shift-operator algebra $\mathcal{A}$, for $n \in \mathbb{N}_{0},(e, k) \in \mathbb{N}_{0}^{ \pm}$.

For any semicircular elements $\Theta_{p, j} \in \mathcal{X}$ of $\mathbb{L} \mathbb{S}$, one has that 


$$
\sigma\left(\Theta_{p, j}\right)^{l}=\sigma\left(\Theta_{p, j}^{l}\right), \text { and } \sigma^{*}\left(\Theta_{p, j}\right)^{l}=\sigma^{*}\left(\Theta_{p, j}^{l}\right),
$$

for all $l \in \mathbb{N}$, since $\sigma$ is a free-homomorphism, and $\sigma^{*}$ is a $*$-homomorphism in $\operatorname{Hom}(\mathbb{L} \mathbb{S})$. Thus, one can get that

$$
\begin{aligned}
\tau^{0}\left(\left(t \sigma\left(\Theta_{p, j}\right)\right)^{l}\right)=\tau^{0}\left(t^{l} \sigma\left(\Theta_{p, j}^{l}\right)\right) & \\
& =t^{l} \tau^{0}\left(\sigma\left(\Theta_{p, j}^{l}\right)\right)=t^{l}\left(\omega_{l} c_{\frac{l}{2}}\right),
\end{aligned}
$$

since $\sigma$ is a free-homomorphism on $\mathbb{L} \mathbb{S}$. Similarly,

$$
\begin{aligned}
& \tau^{0}\left(\left(t \sigma^{*}\left(\Theta_{p, j}\right)\right)^{l}\right)=\tau^{0}\left(t^{l} \sigma^{*}\left(\Theta_{p, j}^{l}\right)\right) \\
& =t^{l} \tau^{0}\left(\sigma^{*}\left(\Theta_{p, j}^{l}\right)\right)= \begin{cases}t^{l}\left(\omega_{l} c_{\frac{l}{2}}\right) & \begin{array}{l}
\text { if } \exists q \in \mathcal{P}, \text { s.t., } \\
g^{n}(q)=p \text { in } \mathcal{P}
\end{array} \\
0 & \text { otherwise. }\end{cases}
\end{aligned}
$$

Motivated by (141), we obtain the following distorted semicircular laws on $\mathbb{L} \mathbb{S}$.

Theorem 17. Let $\Theta_{p, j} \in \mathcal{X}$ be a semicircular element of $\mathbb{L} \mathbb{S}$, and let $t \in \mathbb{R}^{\times}$in $\mathbb{C}$. In addition, suppose the prime $p$ from $\Theta_{p, j}$ is the $n_{p}$-th prime of the TOset $\mathcal{P}$ of (67). For any $n \in \mathbb{N}_{0},(e, k) \in \mathbb{N}_{0}^{ \pm}$, we have that:

(i) the element $t \sigma_{(e, k)}^{n}\left(\Theta_{p, j}\right) \in \mathbb{L} \mathbb{S}$ is $t^{2}$-semicircular in $\mathbb{L} \mathbb{S}$.

(ii) if $n<n_{p}$ in $\mathbb{N}_{0}$, then $t \sigma_{(e, k)}^{* n}\left(\Theta_{p, j}\right)$ is $t^{2}$-semicircular in $\mathbb{L} \mathbb{S}$.

(iii) if $n \geq n_{p}$ in $\mathbb{N}_{0}$, then $t \sigma_{(e, k)}^{* n}\left(\Theta_{p, j}\right)$ has the zero free distribution in $\mathbb{L} \mathbb{S}$

Proof. Let $\Theta_{p, j} \in \mathbb{L} \mathbb{S}$ be a semicircular element, where $p$ is the $n_{p}$-th prime in $\mathcal{P}$, for $j \in \mathbb{Z}$.

First, let $W_{1}=t \sigma_{(e, k)}^{n} \in \mathcal{A}$ be a pi-shift operator with $t \in \mathbb{R}^{\times}$. Then, $W_{1}\left(\Theta_{p, j}\right)=t \Theta_{g^{n}}(p)$,jek is self-adjoint in $\mathbb{L S}$, because $t \in \mathbb{R}^{\times}$. In addition, one has that

$$
\tau^{0}\left(\left(W_{1}\left(\Theta_{p, j}\right)\right)^{l}\right)=t^{l}\left(\omega_{l} c_{\frac{l}{2}}\right)=\omega_{l}\left(t^{2}\right)^{\frac{l}{2}} c_{\frac{l}{2}},
$$

for all $l \in \mathbb{N}$. Thus, this self-adjoint operator $W_{1}\left(\Theta_{p, j}\right)$ is $t^{2}$-semicircular in $\mathbb{L} \mathbb{S}$. For example, the statement (i) in Theorem 17 holds true.

Now, let $W_{2}=t \sigma_{(e, k)}^{* n} \in \mathcal{A}$, for $n<n_{p}$ in $\mathbb{N}_{0}$, and $(e, k) \in \mathbb{N}_{0}^{ \pm}$, where $t \in \mathbb{R}^{\times}$in $\mathbb{C}$. Then, for a given semicircular element $\Theta_{p, j}$ in $\mathbb{L} \mathbb{S}$,

$$
W_{2}\left(\Theta_{p, j}\right)=t \sigma_{(e, k)}^{* n}\left(\Theta_{p, j}\right)=t \Theta_{q, j e k} \in \mathbb{L} \mathbb{S},
$$

where $q \in \mathcal{P}$ satisfying $g^{n}(q)=p$. Since $t \in \mathbb{R}^{\times}$, this element $t \Theta_{q, j e k}$ is self-adjoint in $\mathbb{L} \mathbb{S}$. Moreover,

$$
\tau^{0}\left(\left(W^{2}\left(\Theta_{p, j}\right)\right)^{l}\right)=t^{l}\left(\omega_{l} c_{\frac{l}{2}}\right)=\omega_{l}\left(t^{2}\right)^{\frac{l}{2}} c_{\frac{l}{2}}
$$

for all $l \in \mathbb{N}$, since $n<n_{p}$ in $\mathbb{N}_{0}$. Therefore, the statement (ii) in Theorem 17 holds.

Finally, assume that $n \geq n_{p}$ in $\mathbb{N}_{0}$, and $W_{2}$ is given as above as in (ii) in Theorem 17, where $p$ is the $n_{p}$-th prime in $\mathcal{P}$. Then,

$$
W_{2}\left(\Theta_{p, j}\right)=0_{\mathbb{L} \mathbb{S}} \text { in } \mathbb{L} \mathbb{S},
$$

satisfying 


$$
\tau^{0}\left(\left(W_{2}\left(\Theta_{p, j}\right)\right)^{l}\right)=\tau^{0}\left(0_{\mathbb{L} \mathbb{S}}\right)=0,
$$

for all $l \in \mathbb{N}$. It shows that the free distribution of this self-adjoint operator $W_{2}\left(\Theta_{p, j}\right)$ is characterized by the free-moment sequence,

$$
\left(\tau\left(W_{2}\left(\Theta_{p, j}\right)^{l}\right)\right)_{l=1}^{\infty}=(0,0,0, \ldots) .
$$

Thus, this operator $W^{2}\left(\Theta_{p, j}\right)$ has the zero free distribution in $\mathbb{L} \mathbb{S}$. For example, the statement (iii) in Theorem 17 holds true.

The above theorem illustrates how our semicircular law on $\mathbb{L} \mathbb{S}$ is distorted by the action of the pi-shift-operator semigroup $\sum(\mathbb{L S})$. It explains how the pi-shift-operator algebra $\mathcal{A}$ distorts the free probability on the semicircular Adelic filterization $\mathbb{L} \mathbb{S}$.

\section{Discussion}

As we have seen, there exists an interesting type of Banach-space operators acting on the semicircular elements induced by $\left\{\mathbb{Q}_{p}\right\}_{p \in \mathcal{P}}$, deforming their free distributions, the semicircular law. In particular, the operator-theoretic properties of our pi-shift operators are provided in Theorems 9-16; and some deformations of the semicircular law induced by $\left\{\mathbb{Q}_{p}\right\}_{p \in \mathcal{P}}$ are characterized in Theorem 17.

It is interesting that the pi-shift operators actint on our semicircular elements are like the classical, or generalized Toeplitz operators on Hilbert spaces. In future, one may find connections, or differences between pi-shift-like operators on semicircular elements (as newly-introduced Banach-space operators), and Toeplitz operators (as well-known Hilbert-space operators).

Funding: This research received no external funding.

Acknowledgments: The author personally thank the editors and reviewers of the journal, Mathematics, for their efforts and kind helps.

Conflicts of Interest: The authors declare no conflict of interest.

\section{References}

1. Albeverio, S.; Jorgensen, P.E.T.; Paolucci, A.M. Multiresolution Wavelet Analysis of Integer Scale Bessel Functions. J. Math. Phys. 2007, 48, 073516. [CrossRef]

2. Connes, A. Noncommutative Geometry; Academic Press: San Diego, CA, USA, 1994; ISBN 0-12-185860-X.

3. Connes, A. Trace Formula in Noncommutative Geometry and the Zeroes of the Riemann Zeta Functions. Available online: http:/ / www.alainconnes.org/en/download.php (accessed on 27 April 2019).

4. Gillespie, T. Superposition of Zeroes of Automorphic L-Functions and Functoriality. Ph.D. Thesis, The University of Iowa, Iowa City, IA, USA, 2010.

5. Gillespie, T. Prime Number Theorems for Rankin-Selberg L-Functions over Number Fields. Sci. China Math. 2011, 54, 35-46. [CrossRef]

6. Jorgensen, P.E.T.; Paolucci, A.M. Markov Measures and Extended Zeta Functions. J. Appl. Math. Comput. 2012, 38, 305-323. [CrossRef]

7. Jorgensen, P.E.T.; Paolucci, A.M. States on the Cuntz Algebras and p-Adic Random Walks. J. Aust. Math. Soc. 2011, 90, 197-211. [CrossRef]

8. Cho, I. Semicircular Families in Free Product Banach $*$-Algebras Induced by $p$-Adic Number Fields over Primes p. Complex Anal. Oper. Theory 2017, 11, 507-565. [CrossRef]

9. Cho, I. $p$-Adic Free Stochastic Integrals for $p$-Adic Weighted-Semicircular Motions Determined by Primes $p$. Lib. Math. 2016, 36, 65-110. 
10. Cho, I.; Jorgensen, P.E.T. Semicircular Elements Induced by p-Adic Number Fields. Opusc. Math. 2017, 35, 665-703. [CrossRef]

11. Nica, A.; Speicher, R. Lectures on the Combinatorics of Free Probability, 1st ed.; London Mathematical Society Lecture Note Series; Cambridge University Press: Cambridge, UK, 2006; Volume 335, ISBN 978-0521858526.

12. Speicher, R. Combinatorial Theory of the Free Product with Amalgamation and Operator-Valued Free Probability Theory; American Mathematical Society: Providence, RI, USA, 1998; Volume 132.

13. Speicher, R. A Conceptual Proof of a Basic Result in the Combinatorial Approach to Freeness. Infin. Diment. Anal. Quant. Probab. Relat. Top. 2000, 3, 213-222. [CrossRef]

14. Voiculescu, D. Aspects of Free Analysis. Jpn. J. Math. 2008, 3, 163-183. [CrossRef]

15. Voiculescu, D.; Dykema, K.; Nica, A. Free Random Variables; CRM Monograph Series; American Mathematical Society: Providence, RI, USA, 1992; Volume 1, ISBN 978-0821811405.

16. Cho, I.; Jorgensen, P.E.T. Primes in Intervals and Semicircular Elements Induced by $p$-Adic Analysis on $p$-Adic Number Fields over Primes p. Mathematics 2019, 7, 199. [CrossRef]

17. Radulescu, F. Random Matrices, Amalgamated Free Products and Subfactors of the C*-Algebra of a Free Group of Nonsingular Index. Invent. Math. 1994, 115, 347-389. [CrossRef]

18. Radulescu, F. Free Group Factors and Hecke Operators; Theta Advanced Series in Mathematics; Theta Foundation: Indianapolis, IN, USA, 2014

19. Voiculescu, D. Free Probability and the Von Neumann Algebras of Free Groups. Rep. Math. Phys. 2005, 55, 127-133. [CrossRef]

20. Alpay, D.; Jorgensen, P.E.T. Spectral Theory for Gaussian Processes: Reproducing Kernels. Random Funct. Oper. Theory 2015, 83, 211-229.

21. Alpay, D.; Jorgensen, P.E.T.; Salomon, G. On Free Stochastic Processes and Their Derivatives. Stoch. Process. Appl. 2014, 124, 3392-3411. [CrossRef]

22. Cho, I. Adelic Analysis and Functional Analysis on the Finite Adele Ring. Opusc. Math. 2017, 38, $139-185$. [CrossRef]

23. Cho, I. Semicircular-Like and Semicircular Laws on Banach $*$-Probability Spaces Induced by Dynamical Systems of the Finite Adele Ring. Adv. Oper. Theory 2018, 4, 24-70. [CrossRef]

24. Vladimirov, V.S.; Volovich, I.V.; Zelenov, E.I. p-Adic Analysis and Mathematical Physics; Series on Soviet \& East European Mathematics; World Scientific: Singapore, 1994; Volume 1, ISBN 978-981-02-0880-6.

25. Vladimirov, V.S. p-Adic Quantum Mechanics. Commun. Math. Phys. 1989, 123, 659-676. [CrossRef]

(C) 2019 by the authors. Licensee MDPI, Basel, Switzerland. This article is an open access article distributed under the terms and conditions of the Creative Commons Attribution (CC BY) license (http:/ / creativecommons.org/licenses/by/4.0/). 